\title{
Effect of forced harmonic vibration pile to its adjacent pile in layered elastic soil with double-shear model
}

\author{
Jue Wang ${ }^{\mathrm{a}}$, S. H. Lo ${ }^{\mathrm{b}}$, Ding Zhou ${ }^{\mathrm{a}}$ \\ ${ }^{a}$ College of Civil Engineering, Nanjing University of Technology, Nanjing, China \\ ${ }^{\mathrm{b}}$ Department of Civil Engineering, the University of Hong Kong, Hong Kong, China
}

\begin{abstract}
A new model named double-shear model based on Pasternak foundation and Timoshenko beam theory is developed to determine the dynamic interaction factors for adjacent piles in multilayered soil medium. The double-shear model takes into account the shear deformation and the rotational inertia of piles as well as the shear deformation of soil. Piles are simulated as Timoshenko beams embedded in a layered Pasternak foundation. The differential equation of transverse vibration for a pile is solved by the initial parameter method. The pile-to-pile dynamic interaction factors for the layered soil medium are obtained by the transfer matrix method. The formulation and the implementation have been verified by means of simplified examples. The individual shear effects of soil and pile on the interaction factors are evaluated through a parametric study. Compared to Winkler model with Euler beam, the new model gives much better results for the dynamic interaction of piles embedded in stiff soil with small length-to-diameter ratios. Finally, a detailed study of the dynamic interaction between adjacent piles with different lengths embedded in multilayered soil medium has also been done.
\end{abstract}

Keywords: dynamic interaction factor; Pasternak foundation; Timoshenko beam; layered soils. 


\section{Introduction}

Piles as deep foundations have been commonly used to support engineering structures in the form of closely spaced group. In addition to the loads transmitting from the pile cap, each pile in the group would experience additional loads arising from the interference of adjacent piles. For statically loaded piles, Poulos [1] introduced the concept of 'interaction factor', which is equal to the ratio of displacement of an unloaded pile to that of a loaded pile due to soil deformation. In practice, piles of different lengths can be used to improve their performance and provide a more economical solution. Attention has been paid to the interaction factors for piles of unequal lengths [2-4]. Wong and Poulos [2] developed an approximate solution for the settlement interaction factor between two piles of different diameters or lengths in homogenous medium by the boundary element method. It was extended later by Zhangs [3] to a layered soil medium through the shear displacement method. Liang et al. [4] adopted an integral equation method with a fictitious pile model to analyze the piled raft foundation supported by piles of unequal lengths.

The aforementioned static interaction factors are not applicable to the dynamic analysis of piles, except perhaps at very low frequencies of oscillation. Kaynia and Kausel [5] extended the pile-to-pile interaction factor for identical piles to the dynamic analysis by the boundary integral techniques. Nowadays, various methods, such as the experimental method [6], the finite element method [7, 8], the boundary element method [9-11] and the analytical method [12-15] have been developed for studying the pile-to-pile interaction under dynamic excitation. By the analytical method, the pile is simulated as a Bernoulli-Euler beam imbedded in a Winkler foundation. Due to its clear physical concept and low computational complexity, this method has received widespread 
applications. By means of a simplified wave interference analysis, Mylonakis and Gazetas [16, 17] determined pile-to-pile interaction factor for axial and lateral vibration on piles in homogeneous or two-layer soil based on a Winkler foundation. Using this simplified model, Huang et al. [18] evaluated the pile-to-pile interaction factor for the multilayered foundation. The method was further refined to show the influence of an additional axial load on the interaction factors between piles by Jiang and Song [19]. Hasan and Mehraz [20] analyzed the interaction factors between two adjacent piles with an inclination angle.

However, by the Winkler foundation, the soil pressure at any point is assumed to be proportional only to the deflection at that point. Therefore, it cannot represent the real situation of continuous deformation of the soil medium. To overcome this limitation, the Pasternak foundation [21, 22] has been introduced to include the shear effect of soil. Rosa and Maurizi [23, 24] investigated the vibration frequencies of a beam and multistep pile based on the Pasternak foundation. Qetin and Simsek [25] studied the free vibration of a graded pile embedded in uniform Pasternak foundation and analyzed the variation of the non-dimensional frequency of the pile with respect to the two elastic parameters. Their works have been done within the scope of classical Bernoulli-Euler theory to investigate the dynamic characteristics of piles for mathematical simplicity. It is well known that only the lateral inertia and the elastic forces caused by bending deflections are considered in the Euler theory. On the other hand, for those piles with small length-to-diameter ratio or piles under high frequency excitations, the Timoshenko beam theory [26-29], which takes into account the effects of shear deformation and rotational inertia, may give a better approximation to the general behavior of the piles. 
Following the work of Mylonakis and Gazetas [17], the double-shear model introduced in this paper will be applied to analyze the dynamic interaction of adjacent piles in multilayered soil medium. A parametric study is focused on the shear effects of soil and pile as well as the effect of rotary inertia on the pile-to-pile dynamic interaction factors.

\section{Model for analysis}

To better understand the behavior of dynamic interaction factor for adjacent piles with the same material property but different lengths, the double-shear model to be developed is shown in Fig. 1. The Pasternak foundation is used to represent the reaction of the soil against the pile deformation. Along the pile-shaft, it is a system of infinitely close linear springs and dashpots, which are connecting through an incompressible shear layer. The current methods for determining the parameters of these mechanical components can be classified into experimental method and simplified theoretical formulations $[13,22,30]$. The Timoshenko beam theory is used to describe the transverse vibration of the pile taking into account the shear deformation and the rotational inertia. According to the specific distribution of the soil medium, the source pile and the receiver pile are divided into a number of segments within each soil layer such that the soil within each segment has more or less uniform mechanical properties.

\section{Formulation}

\subsection{Vibration of the source pile}

By using Hamilton principle and the Timoshenko beam theory, the translational and rotational equilibrium conditions of the $i$-th segment of the source pile (Fig. 1b) are given by 


$$
\begin{aligned}
& \rho_{\mathrm{pi}} A_{\mathrm{p} i} \frac{\partial^{2} u_{1 i}(z, t)}{\partial t^{2}}=-\frac{\partial}{\partial z}\left[\kappa G_{\mathrm{p} i} A_{\mathrm{p} i}\left(\theta_{1 i}(z, t)-\frac{\partial u_{1 i}(z, t)}{\partial z}\right)\right]-\left(k_{x i} u_{1 i}(z, t)-g_{x i} \frac{\partial^{2} u_{1 i}(z, t)}{\partial x^{2}}+c_{x i} \frac{\partial u_{1 i}(z, t)}{\partial t}\right) \\
& \rho_{\mathrm{p} i} I_{\mathrm{p} i} \frac{\partial^{2} \theta_{1 i}(z, t)}{\partial t^{2}}=E_{\mathrm{p} i} I_{\mathrm{p} i} \frac{\partial^{2} \theta_{1 i}(z, t)}{\partial \mathrm{z}^{2}}-\kappa G_{\mathrm{p} i} A_{\mathrm{p} i}\left(\theta_{1 i}(z, t)-\frac{\partial u_{1 i}(z, t)}{\partial z}\right)
\end{aligned}
$$

where $A_{\mathrm{p} i}, I_{\mathrm{p} i}$ and $\kappa=6\left(1+v_{\mathrm{p}}\right) /\left(7+6 v_{\mathrm{p}}\right)$ are the area, inertia moment and shear coefficient [26] of the $i$-th segment of pile cross-section, $\rho_{\mathrm{p} i}, E_{\mathrm{p} i}, G_{\mathrm{p} i}, u_{1 i}$ and $\theta_{1 i}$ represent the mass density, Young's modulus, shear modulus, the lateral deflection and the bending rotation of the $i$-th segment of the pile, respectively. The compressive stiffness, the shear stiffness and the damping ratio of the $i$-th segment are given by $k_{x i}=1.2 E_{\mathrm{si}}, g_{x i}=d G_{\mathrm{si}}$ and $c_{x i}=6 a_{0}^{-1 / 4} \rho_{\mathrm{si}} V_{\mathrm{si}} d+2 \beta_{\mathrm{si}} k_{x i} / \omega$.

For the steady-state response, the state variable transformation can be expressed as follows

$u_{1 i}(z, t)=U_{1 i}(z) e^{\mathrm{j} \omega t}, \quad \theta_{1 i}(z, t)=\Theta_{1 i}(z) e^{\mathrm{j} \omega t}$

where $j=\sqrt{-1}, \omega$ is the frequency of the excitation.

Apply the differentiation chain rule on Eqs. (1) and (2), the differential equation of vibration is given by

$$
\begin{aligned}
& E_{\mathrm{p} i} I_{\mathrm{p} i}\left(1+\frac{g_{x i}}{\kappa G_{\mathrm{p} i} A_{\mathrm{p} i}}\right) \frac{\mathrm{d}^{4} U_{1 i}}{\mathrm{~d} z^{4}}+E_{\mathrm{p} i} I_{\mathrm{p} i}\left[\frac{\rho_{\mathrm{p} i} A_{\mathrm{p} i} \omega^{2}-k_{x i}-\mathrm{j} \omega C_{x i}}{\kappa G_{\mathrm{p} i} A_{\mathrm{p} i}}-\frac{g_{x i}}{E_{\mathrm{p} i} I_{\mathrm{p} i}}+\frac{\rho_{\mathrm{p} i} I_{\mathrm{p} i} \omega^{2}}{E_{\mathrm{p} i} I_{\mathrm{p} i}}\left(1+\frac{g_{x i}}{\kappa G_{\mathrm{p} i} A_{\mathrm{p} i}}\right)\right] \frac{\mathrm{d}^{2} U_{1 i}}{\mathrm{~d} z^{2}}- \\
& \left(1-\frac{\rho_{\mathrm{p} i} I_{\mathrm{p} i} \omega^{2}}{\kappa G_{\mathrm{p} i} A_{\mathrm{p} i}}\right)\left(\rho_{\mathrm{p} i} A_{\mathrm{p} i} \omega^{2}-k_{x i}-\mathrm{j} \omega C_{x i}\right) U_{1 i}=0
\end{aligned}
$$

Eq. (4) can also be rewritten in the form

$$
\frac{\mathrm{d}^{4} U_{1 i}}{\mathrm{~d} z^{4}}+\frac{\delta_{i}^{2}}{h_{i}^{2}} \frac{\mathrm{d}^{2} U_{1 i}}{\mathrm{~d} z^{2}}-\frac{\lambda_{i}^{4}}{h_{i}^{4}} U_{1 i}=0
$$

with the following parameters having been introduced in Eq. (5)

$$
\left(\frac{\delta_{i}}{h_{i}}\right)^{2}=\frac{E_{\mathrm{p} i} I_{\mathrm{p} i} R_{i}-J_{\mathrm{p} i} g_{x i}+W_{\mathrm{p} i}\left(J_{\mathrm{p} i}+g_{x i}\right)}{E_{\mathrm{p} i} I_{\mathrm{p} i}\left(J_{\mathrm{p} i}+g_{x i}\right)},\left(\frac{\lambda_{i}}{h_{i}}\right)^{4}=\frac{R_{i}\left(J_{\mathrm{p} i}-W_{\mathrm{p} i}\right)}{E_{\mathrm{p} i} I_{\mathrm{p} i}\left(J_{\mathrm{p} i}+g_{x i}\right)}
$$


where

$J_{\mathrm{p} i}=\kappa G_{\mathrm{p} i} A_{\mathrm{p} i}, \quad W_{\mathrm{p} i}=\rho_{\mathrm{pi}} I_{\mathrm{p} i} \omega^{2}, \quad R_{i}=\rho_{\mathrm{pi}} A_{\mathrm{p} i} \omega^{2}-k_{x i}-\mathrm{j} \omega C_{x i}$.

The general solution of Eq. (5) is

$U_{1 i}(z)=A_{1 i} \cosh \frac{\alpha_{i}}{h_{i}} z+B_{1 i} \sinh \frac{\alpha_{i}}{h_{i}} z+C_{1 i} \cos \frac{\beta_{i}}{h_{i}} z+D_{1 i} \sin \frac{\beta_{i}}{h_{i}} z$

where $\alpha_{i}=\sqrt{\sqrt{\frac{\delta_{i}^{4}}{4}+\lambda_{i}^{4}}-\frac{\delta_{i}^{2}}{2}}$ and $\beta_{i}=\sqrt{\frac{\delta_{i}^{2}}{2}+\sqrt{\frac{\delta_{i}^{4}}{4}+\lambda_{i}^{4}}}, A_{1 i}, B_{1 i}, C_{1 i}, D_{1 i}$ are unknown coefficients which can be determined by boundary conditions at the head and the tip of the pile.

The flexural rotation $\Theta_{1 i}(z)$ at any section of the pile can be determined from Eqs. (1) and (2).

The bending moment $M_{1 i}(z)$ and the shear force $Q_{1 i}(z)$ are related to $U_{1 i}(z)$ and $\Theta_{1 i}(z)$ by

$\Theta_{1 i}=\frac{E_{\mathrm{p} i} I_{\mathrm{p} i}\left(J_{\mathrm{p} i}+g_{x i}\right)}{J_{\mathrm{p} i}\left(J_{\mathrm{p} i}-W_{\mathrm{p} i}\right)} \frac{\mathrm{d}^{3} U_{1 i}}{\mathrm{~d} z^{3}}+\frac{1}{J_{\mathrm{p} i}\left(J_{\mathrm{p} i}-W_{\mathrm{p} i}\right)}\left(E_{\mathrm{p} i} I_{\mathrm{p} i} R_{i}+J_{\mathrm{p} i}{ }^{2}\right) \frac{\mathrm{d} U_{1 i}}{\mathrm{~d} z}$

$M_{1 i}=E_{\mathrm{p} i} I_{\mathrm{p} i} \frac{\mathrm{d} \Theta_{1 i}}{\mathrm{~d} z}$

$Q_{1 i}=J_{\mathrm{p} i}\left(\frac{\mathrm{d} U_{1 i}}{\mathrm{~d} z}-\Theta_{1 i}\right)$

It is remarked that setting $g_{x i}=0$ the equation of motion of a Timoshenko pile on the Winkler foundation is readily obtained, and the case of the Euler pile on elastic soil is reproduced by setting $1 / J_{\mathrm{pi}} \rightarrow 0$ and $W_{\mathrm{p} i} \rightarrow 0$.

The local coordinate system for the $i$-th segment is shown in Fig. 1(b). Based on Eqs. (7-10), the deflections and internal forces of the pile can be written in a matrix form with undetermined coefficients, as shown below

$$
\left\{\begin{array}{c}
U_{1 i}(z) \\
\Theta_{1 i}(z) \\
Q_{1 i}(z) \\
M_{1 i}(z)
\end{array}\right\}=[\boldsymbol{t a}]_{i}\left\{\begin{array}{c}
A_{1 i} \\
B_{1 i} \\
C_{1 i} \\
D_{1 i}
\end{array}\right\}
$$


where the expressions of each elements in matrix $[\boldsymbol{t a}]_{i}$ are given in Appendix A.

From Eq. (11) the four coefficients $A_{1 i}, B_{1 i}, C_{1 i}, D_{1 i}$ can be determined using deflections at the top and at the bottom

$$
\begin{aligned}
& \left\{\begin{array}{l}
A_{1 i} \\
B_{1 i} \\
C_{1 i} \\
D_{1 i}
\end{array}\right\}=\left.[\boldsymbol{t a}]_{i}^{-1}\right|_{z=0}\left\{\begin{array}{c}
U_{1 i}(0) \\
\Theta_{1 i}(0) \\
Q_{1 i}(0) \\
M_{1 i}(0)
\end{array}\right\}=\left.[\boldsymbol{t a}]_{i}^{-1}\right|_{z=h_{i}}\left\{\begin{array}{c}
U_{1 i}\left(h_{i}\right) \\
\Theta_{1 i}\left(h_{i}\right) \\
Q_{1 i}\left(h_{i}\right) \\
M_{1 i}\left(h_{i}\right)
\end{array}\right\} \\
& \Rightarrow\left\{\begin{array}{c}
U_{1 i}\left(h_{i}\right) \\
\Theta_{1 i}\left(h_{i}\right) \\
Q_{1 i}\left(h_{i}\right) \\
M_{1 i}\left(h_{i}\right)
\end{array}\right\}=\left.\left.[\mathbf{t a}]_{i}\right|_{z=h_{i}}[\boldsymbol{t a}]_{i}^{-1}\right|_{z=0}\left\{\begin{array}{c}
U_{1 i}(0) \\
\Theta_{1 i}(0) \\
Q_{1 i}(0) \\
M_{1 i}(0)
\end{array}\right\}
\end{aligned}
$$

Based on the continuity condition at the interface of the adjacent segments, i.e. $\left\{U_{1 i+1}(0), \Theta_{1 i+1}(0), Q_{1 i+1}(0), M_{1 i+1}(0)\right\}=\left\{U_{1 i}\left(h_{i}\right), \Theta_{1 i}\left(h_{i}\right), Q_{1 i}\left(h_{i}\right), M_{1 i}\left(h_{i}\right)\right\}$, the relationship of the deflection and internal force between the head and the tip of the source pile can be given by

$$
\left\{\begin{array}{l}
U_{1}(L) \\
\Theta_{1}(L) \\
Q_{1}(L) \\
M_{1}(L)
\end{array}\right\}=[\mathbf{T A}]\left\{\begin{array}{c}
U_{1}(0) \\
\Theta_{1}(0) \\
Q_{1}(0) \\
M_{1}(0)
\end{array}\right\}
$$

where the transfer matrix $[\mathbf{T A}]=\prod_{i=1}^{N_{1}}[\mathbf{T a}]_{i},[\mathbf{T a}]_{i}=\left.\left.[\boldsymbol{t a}]_{i}\right|_{z=h_{i}}[\boldsymbol{t a}]_{i}^{-1}\right|_{z=0}, N_{1}$ is the number of the segment of the source pile.

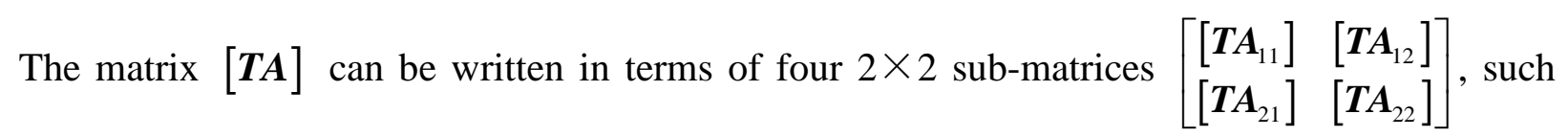
that, Eq. (14) can be expressed as

$$
\left\{\begin{array}{c}
Q_{1}(L) \\
M_{1}(L)
\end{array}\right\}=\left[\boldsymbol{T A}_{21}\right]\left\{\begin{array}{c}
U_{1}(0) \\
\Theta_{1}(0)
\end{array}\right\}+\left[\boldsymbol{T A}_{22}\right]\left\{\begin{array}{c}
Q_{1}(0) \\
M_{1}(0)
\end{array}\right\}
$$

At the tip of the pile, the bending moment and the shear force are assumed to be negligible. By applying this boundary condition, the force-displacement relationship at the pile head can be 
obtained based on Eq. (15),

$$
\left\{\begin{array}{c}
Q_{1}(0) \\
M_{1}(0)
\end{array}\right\}=[\Re]\left\{\begin{array}{l}
U_{1}(0) \\
\Theta_{1}(0)
\end{array}\right\}
$$

where the impedance of a single pile $[\mathfrak{R}]=-\left[\boldsymbol{T A}_{22}\right]^{-1}\left[\boldsymbol{T A}_{21}\right]$.

\subsection{Vibration of the receiver pile}

According to the model developed by Dobry and Gazetas [14] as shown in Fig. 2, at the distance $s$ from the oscillating pile and an angel $\phi$ from the direction of loading, the displacement field can be expressed as

$$
\begin{aligned}
& u_{s i}(z, t)=U_{s i}(z) e^{\mathrm{j} \omega t}=\varphi_{i}(s, \phi) U_{1 i}(z) e^{\mathrm{j} \omega t} \\
& \varphi_{i}(s, 0)=\sqrt{\frac{d}{2 s}} \exp \left[\frac{-\omega\left(\beta_{\mathrm{si}}+\mathrm{j}\right)(s-d / 2)}{V_{\mathrm{La} i}}\right] \\
& \varphi_{i}\left(s, \frac{\pi}{2}\right)=\sqrt{\frac{d}{2 s}} \exp \left[\frac{-\omega\left(\beta_{\mathrm{si}}+\mathrm{j}\right)(s-d / 2)}{V_{\mathrm{si}}}\right] \\
& \varphi_{i}(s, \phi)=\varphi_{i}(s, 0) \cos ^{2} \phi+\varphi_{i}\left(s, \frac{\pi}{2}\right) \sin ^{2} \phi
\end{aligned}
$$

where $V_{\mathrm{si}}=\sqrt{\frac{E_{\mathrm{si}}}{2\left(1+v_{\mathrm{si}}\right) \rho_{\mathrm{si}}}}$ is the shear wave velocity; $E_{\mathrm{s} i}, v_{\mathrm{s} i}, \beta_{\mathrm{s} i}$ and $\rho_{\mathrm{si}}$ are the Young's modulus, the Poisson's ratio, the radiation damping and the mass density of $i$-th soil layer, respectively; $V_{\mathrm{Lai}}=\frac{3.4 V_{\mathrm{si}}}{\pi\left(1-v_{\mathrm{si}}\right)}$ is the Lysmer's analog wave velocity. $\varphi_{i}(s, \phi)$ is the attenuation function, $\phi$ is the angle between the centre-line direction of two piles and the direction of the applied horizontal force, $s$ is the center to center distance between two piles.

Utilizing the Timoshenko beam theory and the Pasternak foundation, with the consideration of the dynamic interaction between the soil and the receiver pile, the translation and rotational equilibrium conditions of the $i$-th segment at the receiver pile are given by 


$$
\begin{aligned}
& \rho_{\mathrm{pi}} A_{\mathrm{p} i} \frac{\partial^{2} u_{2 i}}{\partial t^{2}}=-\frac{\partial}{\partial z}\left[\kappa G_{\mathrm{p} i} A_{\mathrm{p} i}\left(\theta_{2 i}-\frac{\partial u_{2 i}}{\partial z}\right)\right]-\left[k_{x i}\left(u_{2 i}-u_{s i}\right)-g_{x i}\left(\frac{\partial^{2} u_{2 i}}{\partial z^{2}}-\frac{\partial^{2} u_{s i}}{\partial \mathrm{z}^{2}}\right)+c_{x i}\left(\frac{\partial u_{2 i}}{\partial t}-\frac{\partial u_{s i}}{\partial t}\right)\right] \\
& \rho_{\mathrm{p} i} I_{\mathrm{p} i} \frac{\partial^{2} \theta_{2 i}}{\partial t^{2}}=E_{\mathrm{p} i} I_{\mathrm{p} i} \frac{\partial^{2} \theta_{2 i}}{\partial \mathrm{z}^{2}}-\kappa G_{\mathrm{p} i} A_{\mathrm{p} i}\left(\theta_{2 i}-\frac{\partial u_{2 i}}{\partial z}\right)
\end{aligned}
$$

Eliminating $\theta_{2 i}$ from Eqs. (18) and (19), we have the following fourth-order differential equation for the steady-state response

$$
\begin{aligned}
& E_{\mathrm{p} i} I_{\mathrm{p} i}\left(1+\frac{g_{x i}}{\kappa G_{\mathrm{p} i} A_{\mathrm{p} i}}\right) \frac{\mathrm{d}^{4} U_{2 i}}{\mathrm{~d} z^{4}}+E_{\mathrm{p} i} I_{\mathrm{p} i}\left[\frac{\rho_{\mathrm{p} i} A_{\mathrm{p} i} \omega^{2}-k_{x i}-\mathrm{j} \omega c_{x i}}{\kappa G_{\mathrm{p} i} A_{\mathrm{p} i}}-\frac{g_{x i}}{E_{\mathrm{p} i} I_{\mathrm{p} i}}+\frac{\rho_{\mathrm{p} i} I_{\mathrm{p} i} \omega^{2}}{E_{\mathrm{p} i} I_{\mathrm{p} i}}\left(1+\frac{g_{x i}}{\kappa G_{\mathrm{p} i} A_{\mathrm{p} i}}\right)\right] \frac{\mathrm{d}^{2} U_{2 i}}{\mathrm{~d} z^{2}}-\left(1-\frac{\rho_{\mathrm{p} i} I_{\mathrm{p} i} \omega^{2}}{\kappa G_{\mathrm{p} i} A_{\mathrm{p} i}}\right) \times \\
& \left(\rho_{\mathrm{p} i} A_{\mathrm{p} i} \omega^{2}-k_{x i}-\mathrm{j} \omega C_{x i}\right) U_{2 i}=\left(1-\frac{\rho_{\mathrm{p} i} I_{\mathrm{pi}} \omega^{2}}{\kappa G_{\mathrm{p} i} A_{\mathrm{p} i}}\right)\left(k_{x i}+\mathrm{j} \omega C_{x i}\right) U_{s i}-\frac{E_{\mathrm{p} i} I_{\mathrm{p} i}\left(k_{x i}+\mathrm{j} \omega C_{x i}\right)-\rho_{\mathrm{p} i} I_{\mathrm{p} i} \omega^{2} g_{x i}}{\kappa G_{\mathrm{p} i} A_{\mathrm{p} i}} \frac{\mathrm{d}^{2} U_{s i}}{\mathrm{dz}}+\frac{E_{\mathrm{p} i} I_{\mathrm{p} i} g_{x i}}{\kappa G_{\mathrm{pi}} A_{\mathrm{p} i}} \frac{\mathrm{d}^{4} U_{s i}}{\mathrm{dz}}
\end{aligned}
$$

Following the notations of Eq. (6), Eq. (20) can be rewritten as

$$
\frac{\mathrm{d}^{4} U_{2 i}}{\mathrm{dz} z^{4}}+\frac{\delta_{i}^{2}}{h_{i}^{2}} \frac{\mathrm{d}^{2} U_{2 i}}{\mathrm{~d} z^{2}}-\frac{\lambda_{i}^{4}}{h_{i}^{4}} U_{2 i}=f_{a i}(s, \phi) U_{1 i}+f_{b i}(s, \phi) \frac{\mathrm{d}^{2} U_{1 i}}{\mathrm{~d} z^{2}}+f_{c i}(s, \phi) \frac{\mathrm{d}^{4} U_{1 i}}{\mathrm{dz}^{4}}
$$

with the following parameters having been introduced

$$
\begin{aligned}
& f_{a i}=\frac{\left(J_{\mathrm{p} i}-W_{\mathrm{p} i}\right)\left(k_{x i}+\mathrm{j} \omega c_{x i}\right)}{E_{\mathrm{p} i} I_{\mathrm{p} i}\left(J_{\mathrm{p} i}+g_{x i}\right)} \psi_{i}(s, \phi) ; \\
& f_{b i}=\frac{E_{\mathrm{p} i} I_{\mathrm{p} i}\left(k_{x i}+\mathrm{j} \omega c_{x i}\right)-W_{\mathrm{p} i} g_{x i}}{E_{\mathrm{p} i} I_{\mathrm{p} i}\left(J_{\mathrm{p} i}+g_{x i}\right)} \psi_{i}(s, \phi) ; \\
& f_{c i}=\frac{g_{x i}}{J_{\mathrm{p} i}+g_{x i}} \psi_{i}(s, \phi) .
\end{aligned}
$$

The general solution of Eq. (21) is given by

$$
U_{2 i}^{*}(z)=A_{2 i} \cosh \frac{\alpha_{i}}{h_{i}} z+B_{2 i} \sinh \frac{\alpha_{i}}{h_{i}} z+C_{2 i} \cos \frac{\beta_{i}}{h_{i}} z+D_{2 i} \sin \frac{\beta_{i}}{h_{i}} z
$$

where $A_{2 i}, B_{2 i}, C_{2 i}, D_{2 i}$ are unknown coefficients to be determined from the boundary conditions of the receiver pile. 
The particular solution of Eq. (21) is

$$
U_{2 i}^{* *}(z)=z F_{a i}\left[A_{1 i} \sinh \left(\frac{\alpha_{i}}{h_{i}} z\right)+B_{1 i} \cosh \left(\frac{\alpha_{i}}{h_{i}} z\right)\right]+z F_{b i}\left[-C_{1 i} \sin \left(\frac{\beta_{i}}{h_{i}} z\right)+D_{1 i} \cos \left(\frac{\beta_{i}}{h_{i}} z\right)\right]
$$

where

$$
F_{a i}=\frac{f_{a i}+f_{b i}\left(\frac{\alpha_{i}}{h_{i}}\right)^{2}+f_{c i}\left(\frac{\alpha_{i}}{h_{i}}\right)^{4}}{2\left(\frac{\alpha_{i}}{h_{i}}\right)\left[2\left(\frac{\alpha_{i}}{h_{i}}\right)^{2}+\left(\frac{\delta_{i}}{h_{i}}\right)^{2}\right]}, F_{b i}=\frac{f_{a i}-f_{b i}\left(\frac{\beta_{i}}{h_{i}}\right)^{2}+f_{c i}\left(\frac{\beta_{i}}{h_{i}}\right)^{4}}{2\left(\frac{\beta_{i}}{h_{i}}\right)\left[2\left(\frac{\beta_{i}}{h_{i}}\right)^{2}-\left(\frac{\delta_{i}}{h_{i}}\right)^{2}\right]}
$$

Hence, the solution of Eq. (21) can be expression as

$$
U_{2 i}(z)=U_{2 i}^{*}(z)+U_{2 i}^{* *}(z)
$$

Similar to the source pile in section 2.1, the deflections and internal forces of the receiver pile can be expressed in a matrix form with undetermined coefficients

$$
\left\{\begin{array}{l}
U_{2 i}(z) \\
\Theta_{2 i}(z) \\
Q_{2 i}(z) \\
M_{2 i}(z)
\end{array}\right\}=[\boldsymbol{t a}]_{i}\left\{\begin{array}{l}
A_{2 i} \\
B_{2 i} \\
C_{2 i} \\
D_{2 i}
\end{array}\right\}+[\boldsymbol{t b}]_{i}\left\{\begin{array}{l}
A_{1 i} \\
B_{1 i} \\
C_{1 i} \\
D_{1 i}
\end{array}\right\}
$$

where the expression for the elements in matrices $[\boldsymbol{t a}]_{i}$ and $[\boldsymbol{t} \boldsymbol{b}]_{i}$ are shown in Appendix A and B.

Substituting Eq. (12) into Eq. (25), the unknown coefficients can be expressed as

$$
\left\{\begin{array}{c}
A_{2 i} \\
B_{2 i} \\
C_{2 i} \\
D_{2 i}
\end{array}\right\}=\left.[\boldsymbol{t a}]_{i}^{-1}\right|_{z=0}\left(\left\{\begin{array}{c}
U_{2 i}(0) \\
\Theta_{2 i}(0) \\
Q_{2 i}(0) \\
M_{2 i}(0)
\end{array}\right\}-\left.\left.[\boldsymbol{t} \boldsymbol{b}]_{i}\right|_{z=0}[\boldsymbol{t a}]_{i}^{-1}\right|_{z=0}\left\{\begin{array}{c}
U_{1 i}(0) \\
\Theta_{1 i}(0) \\
Q_{1 i}(0) \\
M_{1 i}(0)
\end{array}\right\}\right)
$$

The deflections and internal forces between the top and the bottom of the $i$-th segment of the receiver pile are as follows 
$\left\{\begin{array}{c}U_{2 i}\left(h_{i}\right) \\ \Theta_{2 i}\left(h_{i}\right) \\ Q_{2 i}\left(h_{i}\right) \\ M_{2 i}\left(h_{i}\right)\end{array}\right\}=[\boldsymbol{T a}]_{i}\left\{\begin{array}{c}U_{2 i}(0) \\ \Theta_{2 i}(0) \\ Q_{2 i}(0) \\ M_{2 i}(0)\end{array}\right\}+[\boldsymbol{T} \boldsymbol{b}]_{i}\left\{\begin{array}{c}U_{1 i}(0) \\ \Theta_{1 i}(0) \\ Q_{1 i}(0) \\ M_{1 i}(0)\end{array}\right\}$

where $[\boldsymbol{T} \boldsymbol{b}]_{i}=-\left.\left.\left.\left.[\boldsymbol{t a}]_{i}\right|_{z=h_{i}}[\boldsymbol{t a}]_{i}^{-1}\right|_{z=0}[\boldsymbol{t} \boldsymbol{b}]_{i}\right|_{z=0}[\boldsymbol{t a}]_{i}^{-1}\right|_{z=0}+\left.\left.[\boldsymbol{t} \boldsymbol{b}]_{i}\right|_{z=h_{i}}[\boldsymbol{t a}]_{i}^{-1}\right|_{z=0}$.

Based on the deflection and force continuity condition at the interface of the adjacent segments, i.e. $\left\{U_{2 i+1}(0), \Theta_{2 i+1}(0), Q_{2 i+1}(0), M_{2 i+1}(0)\right\}=\left\{U_{2 i}\left(h_{i}\right), \Theta_{2 i}\left(h_{i}\right), Q_{2 i}\left(h_{i}\right), M_{2 i}\left(h_{i}\right)\right\}$, we have the following the relationship for the deflections and internal forces between the head and the tip of the source pile

$$
\left\{\begin{array}{l}
U_{2}(L) \\
\Theta_{2}(L) \\
Q_{2}(L) \\
M_{2}(L)
\end{array}\right\}=[\boldsymbol{T C}]\left\{\begin{array}{c}
U_{2}(0) \\
\Theta_{2}(0) \\
Q_{2}(0) \\
M_{2}(0)
\end{array}\right\}+[\boldsymbol{T B}]\left\{\begin{array}{c}
U_{1}(0) \\
\Theta_{1}(0) \\
Q_{1}(0) \\
M_{1}(0)
\end{array}\right\}
$$

where the transfer matrix $[\boldsymbol{T C}]=\prod_{j=1}^{N_{2}}[\mathbf{T a}]_{j},[\mathbf{T B}]=\sum_{j=1}^{N_{2}}[\boldsymbol{T a}]_{N_{2}} \cdots[\mathbf{T a}]_{j+1}[\boldsymbol{T} \boldsymbol{b}]_{j}[\mathbf{T a}]_{j-1} \cdots[\mathbf{T a}]_{1}, N_{2}$ is the number of the segment of the receiver pile.

The matrix $[\mathbf{T B}]$ and $[\boldsymbol{T C}]$ can be written in four $2 \times 2$ sub-matrices in the same way as $[\boldsymbol{T A}]$ in section 2.1, and from Eq. (28) we have

$$
\left\{\begin{array}{c}
Q_{2}(L) \\
M_{2}(L)
\end{array}\right\}=\left[\boldsymbol{T C}_{21}\right]\left\{\begin{array}{c}
U_{2}(0) \\
\Theta_{2}(0)
\end{array}\right\}+\left[\boldsymbol{T C}_{22}\right]\left\{\begin{array}{c}
Q_{2}(0) \\
M_{2}(0)
\end{array}\right\}+\left[\boldsymbol{T B}_{21}\right]\left\{\begin{array}{c}
U_{1}(0) \\
\Theta_{1}(0)
\end{array}\right\}+\left[\boldsymbol{T} \boldsymbol{B}_{22}\right]\left\{\begin{array}{c}
Q_{1}(0) \\
M_{1}(0)
\end{array}\right\}
$$

As the moment and the shear at the head and at the tip of the receiver pile are negligible,

$$
\left\{\begin{array}{l}
U_{2}(0) \\
\Theta_{2}(0)
\end{array}\right\}=[\boldsymbol{a}(s, \phi)]\left\{\begin{array}{l}
U_{1}(0) \\
\Theta_{1}(0)
\end{array}\right\}
$$

where $[\boldsymbol{a}(s, \phi)]=-\left[\boldsymbol{T} \boldsymbol{C}_{21}\right]^{-1}\left\{\left[\boldsymbol{T} \boldsymbol{B}_{21}\right]+\left[\boldsymbol{T} \boldsymbol{B}_{22}\right][\mathfrak{R}]\right\}$.

\subsection{Pile-to-pile dynamic interaction factor}

The interaction factor between the adjacent piles is defined as the ratio of the additional displacement at the head of the receiver pile, due to the source pile, to the displacement at the head 
of the source pile. Letting $[\boldsymbol{f}]=[\Re]^{-1}$, the lateral interaction factor $\alpha_{\text {up }}$ and the rocking interaction factor $\alpha_{\theta \mathrm{m}}$ between adjacent piles are given by

$$
\begin{aligned}
& \alpha_{\text {up }}=\frac{U_{2}(0)}{U_{1}(0)}=\frac{\left[\boldsymbol{a}_{1,1}\right]\left[\boldsymbol{f}_{1,1}\right]+\left[\boldsymbol{a}_{1,2}\right]\left[\boldsymbol{f}_{2,1}\right]}{\left[\boldsymbol{f}_{1,1}\right]} \\
& \alpha_{\theta \mathrm{m}}=\frac{\Theta_{2}(0)}{\Theta_{1}(0)}=\frac{\left[\boldsymbol{a}_{2,2}\right]\left[\boldsymbol{f}_{2,2}\right]+\left[\boldsymbol{a}_{2,1}\right]\left[\boldsymbol{f}_{1,2}\right]}{\left[\boldsymbol{f}_{2,2}\right]}
\end{aligned}
$$

\section{Numerical examples and discussions}

\subsection{Comparison study}

If the shear stiffness of the soil $g_{x i}$, the pile parameters $1 / J_{\mathrm{p} i}$ and $W_{\mathrm{p} i}$ are assumed to be zero, then the model is reduced to Euler beam with the Winkler foundation. To validate the formulation and the coding, the reduced results could be compared with those of Gazetas et al. [13, 17]. As illustrated in Fig. 3, the lateral interaction factor $\alpha_{\mathrm{up}}$ and rocking factor $\alpha_{\theta \mathrm{m}}$ in homogeneous foundation obtained by the present study are in agreement with that by Mylonakis and Gazetas [17]. The lateral interaction factor is also compared with the results from Dobry and Gazetas [13] as shown in Fig. 3a. It can be seen from Fig. 3a that the results obtained by the present study are less than those by Dobry and Gazetas as the interaction between the receiver pile and the surrounding soil was neglected. However, the difference decreases for increasing value of the piles distance $(s / d)$ ratio. Fig. 4 refers to a pair of interacting piles in a two-layered soil foundation which consists of a soft surface layer over a homogeneous half-space. The lateral interaction factors are also in good agreement with those of Mylonakis and Gazetas.

\subsection{Shear effect of soil on interaction factor}

We are interested to find out the difference in modeling the soil using the two kinds of 
foundations, Pasternak foundation and Winkler foundation. From the observation of the numerical results in Fig. 5, it can be seen that lateral and rocking interaction factors obtained from the Pasternak foundation are obviously larger than those obtained from the Winkler foundation within the whole range of frequencies examined for $E_{\mathrm{p}} / E_{\mathrm{s}}=100$. However, the difference becomes less significant for the case of $E_{\mathrm{p}} / E_{\mathrm{s}}=1000$. Generally, the lower is the pile-soil elastic modulus ratio, the greater is the difference between the models.

\subsection{Shear effect of pile on interaction factor}

As it is well known that the Timoshenko beam theory takes into account shear deformation and rotational inertia effects, making it more suitable for describing the dynamical behavior of those piles with a low length-to-diameter $(L / d)$ ratio subjected to high-frequency excitations. This example shows the influence of $L / d$ on the lateral interaction factor $\alpha_{\mathrm{up}}$ and the rocking interaction factor $\alpha_{\theta \mathrm{m}}$ for the Timoshenko pile and Euler pile, respectively. Fig. 6 shows a homogenous half-space and Fig. 7 shows a two-layered soil profile, which consists of a soft surface layer over a stiffer half-space. In order to compare with the available results, the shear coefficients of soil $g_{x i}$ in this example are set to 0. It can be seem from Figs. 6 and 7 that the Euler pile approaches the Timoshenko beam for high values of $L / d$ ratio, and lower dynamic interaction $\alpha_{\text {up }}$ and $\alpha_{\theta \mathrm{m}}$ calculated from Timoshenko model than that from Euler one can be observed for small values of $L / d$. The numerical results implies that the difference between the two pile models is less than $5 \%$ when the ratio $L / d$ is larger than 6 , as shown in Figs. 6a, 6b, 7a and 7b. It should be noticed that due to the rotational inertia effect there is a small discrepancy between the results from the Euler and the Timoshenko models in Figs. 6c and 7c, 
even for a large length-to-diameter ratio $L / d=25$. This is due to a higher excitation frequency which makes the effect of the rotational inertia more prominent between the Timoshenko pile and the Euler pile. Therefore, it is recommended to take shear effect into account for the case of piles with a length-to-diameter ratio $L / d<6$ under high frequency excitation.

\subsection{Application to long-short piles}

It can be seen from the previous sections that shear effects of soil and pile may have a significant influence on the interaction between piles. As an extension work of Mylonakis and Gazetas, the presented double-shear model is applied to analyze the interaction factors of a loaded long pile (length $L_{\mathrm{s}}$ ) on an unloaded short pile (length $L_{\mathrm{r}}$ ) embedded in multiple layered soil mediums as shown in Table. 1. Cases 1 and 3 refer to a layered medium covered respectively by a soft layer and a stiff surface layer. Case 2 refers to a layered medium with Young’s modulus increasing linearly with depth. The influence of the length of shorter pile on the pile-to-pile interaction with the same elastic modulus $E_{\mathrm{p}}=38 \mathrm{GPa}$ and equal diameter $d=0.5 \mathrm{~m}$, is analyzed, as shown in Figs. 8 and 9 . It can be observed that the lateral interaction factor $\alpha_{\text {up }}$ (as shown in Fig. 8) and the rocking interaction factor $\alpha_{\theta \mathrm{m}}$ (as shown in Fig. 9) between the two piles of unequal lengths may have a difference up to 25\% when the length ratio $L_{\mathrm{s}} / L_{\mathrm{r}}$ reaches 1:0.8. Comparing of Figs. 8a, 8b and 8c (or Figs. 9a, 9b and 9c), it can be seen that the pile-to-pile interaction factors between long and short piles embedded in a layered medium covered by a soft surface layer could have a more significant difference. 


\section{Conclusion}

The double-shear model in terms of transfer matrix has been presented in this paper to analyze the dynamic interaction of piles in multilayered soil medium. The limiting solutions are in agreement with those computed with Winkler-Euler method by Mylonakis and Gazetas. From the investigation of the effects of shear deformation and rotational inertia of adjacent piles as well as that of soil shear deformation, the following conclusions can be drawn

(i) The double-shear model employing the Pasternak foundation in general gives a better approximation to the behavior of the soil medium, especially for the case of a low pile-soil Young's modulus ratio $\left(E_{\mathrm{p}} / E_{\mathrm{s}}\right)$.

(ii) As shear deformation and rotational inertia are neglected, the difference between the Timoshenko pile and the Euler pile becomes significant when the length-to-diameter $(L / d)$ ratio is small.

(iii) As another advantage over conventional models, the double-shear model is also applicable to the determination of the dynamic interaction factor between piles of unequal lengths, which is commonly encountered in foundation engineering.

\section{Acknowledgements}

The financial supports from the HKSAR GRF Grant (HKU 71511OE) and the Natural Science Foundation of Jiangsu Province, China (SBK201322459) are greatly acknowledged. 


\section{Reference}

[1] Poulos G H. Analysis of the settlement of pile groups [J]. Geotechnique, 1968, 18(4):449-471.

[2] Wong S C, Poulos H G. Approximate pile-to-pile interaction factors between two dissimilar piles [J]. Computers and Geotechnics, 2005, 32(8): 613-618.

[3] Zhang Q, Zhang Z. Study on interaction between dissimilar piles in layered soils [J]. International Journal for Numerical and Analytical Methods in Geomechanics, 2011, 35(1): 67-81.

[4] Liang F, Chen L, Han J. Integral equation method for analysis of piled rafts with dissimilar piles under vertical loading [J]. Computers and Geotechnics, 2009, 36(3): 419-426.

[5] Kaynia A M, Kausel E. Dynamic stiffness and seismic response of pile groups [R]. Res. Rep. No. R82-03, Cambridge: Massachusetts Institute of Technology, 1982.

[6] Dezi F, Gara F, Roia D. Experimental study of near-shore pile-to-pile interaction [J]. Soil Dynamics and Earthquake Engineering, 2013, 48: 282-293.

[7] Wolf J.P, Von Arx G.A. Impedance functions of a group of vertical piles [C]. Proceedings of the ASCE Geotechnical Engineering Division Specialty Conference, Pasadena, California, 1978, 2:1024-1041.

[8] Wu G, Finn W D L. Dynamic elastic analysis of pile foundations using finite element method in the frequency domain [J]. Canadian Geotechnical Journal, 1997, 34(1): 34-43.

[9] Mamoon S M, Kaynia A M, Banerjee P K. Frequency domain dynamic analysis of piles and pile groups[J]. Journal of Engineering Mechanics, 1990, 116(10): 2237-2257.

[10] Maeso O, Aznárez J J, García F. Dynamic impedances of piles and groups of piles in saturated soils[J]. Computers \& structures, 2005, 83(10): 769-782.

[11] Padron L A, Aznarez J J, Maeso O. Dynamic analysis of piled foundations in stratified soils by a BEM-FEM model [J]. Soil Dynamics and Earthquake Engineering, 2008, 28(5): 333-346.

[12] Novak M. Dynamic stiffness and damping of piles [J]. Canadian Geotechnical Journal, 1974, 11(4):574-598.

[13] Dobry R, Gazetas G. Simple method for dynamic stiffness and damping of floating pile groups [J]. Geotechnique, 1988,38(4):557-574.

[14] Gazetas G, Dobry R. Dynamic pile-soil-pile interaction, Part I: Analysis of axial vibration [J]. Earthquake Engineering and Structural Dynamic,1991,20:115-132.

[15] Markris N, Gazetas G. Dynamic pile-soil-pile interaction, Part II: Lateral and seismic response [J]. Earthquake Engineering and Structural Dynamic, 1992,21(2):145-162.

[16] Mylonakis G, Gazetas G. Vertical vibration and additional distress of grouped piles in layered soil [J]. Soils and foundations, 1998, 38(1): 1-14.

[17] Mylonakis G, Gazetas G. Lateral vibration and internal forces of grouped piles in layered soil [J]. Journal of Geotechnical and Geoenvironmental Engineering, 1999, 125(1):16-25.

[18] Huang M S, Wu Z, Ren Q. Lateral vibration of pile groups in layered soil [J]. Chinese Journal of Geotechnical Engineering, 2007, 29(1): 32-38.

[19] Jianguo J, Xuhong Z, Jiasheng Z. Dynamic interaction factor considering axial load [J]. Geotechnical and Geological Engineering, 2007, 25(4): 423-429.

[20] Hasan G, Mehraz A. Pile-soil-pile interaction in pile groups with batter piles under dynamic loads [J]. Soil Dynamics and Earthquake Engineering, 2011, 31(8): 1159-1170.

[21] Pasternak P L. On a new method of analysis of an elastic foundation by means of two foundation constants. Gosudarstvennoe Izdatel’stvo Litearturi po Stroitel’stvu i Arkhitekture, Moscow, USSR (in Russian), 1954. 
[22] Dutta S C, Roy R. A critical review on idealization and modeling for interaction among soil-foundation-structure system [J]. Computers \& Structures, 2002, 80(20): 1579-1594.

[23] De Rosa M A, Maurizi M J. The influence of concentrated masses and Pasternak soil on the free vibrations of Euler beams-exact solution [J]. Journal of Sound and Vibration, 1998, 212(4): 573-581.

[24] De Rosa M A, Maurizi M J. Dynamic analysis of multistep piles on Pasternak soil subjected to axial tip forces [J]. Journal of sound and vibration, 1999, 219(5): 771-783.

[25] Qetin D, Simsek M. Free vibration of an axially functionally graded pile with pinned ends embedded in Winkler-Pasternak elastic medium[J]. Structural Engineering and Mechanics, 2011, 40(4): 583-594.

[26] Timoshenko S P, Gere J M. Mechanics of materials. Van Nostrand Reinhold Co., 1972.

[27] Yokoyama T. Vibration analysis of Timoshenko beam-columns on two-parameter elastic foundations [J]. Computers \& Structures, 1996, 61(6): 995-1007.

[28] Kargarnovin M H, Younesian D. Dynamics of Timoshenko beams on Pasternak foundation under moving load [J]. Mechanics Research Communications, 2004, 31(6): 713-723.

[29] Calio I, Greco A. Free vibrations of Timoshenko beam-columns on Pasternak foundations [J]. Journal of Vibration and Control, 2013, 19(5): 686-696.

[30] Dutta S C, Roy R. A critical review on idealization and modeling for interaction among soil-foundation-structure system[J]. Computers \& Structures, 2002, 80(20): 1579-1594. 
Table 1. The distribution of pile-soil modulus $\left(E_{\mathrm{p}} / E_{\mathrm{s}}\right)$ ratio along the soil thickness

\begin{tabular}{ccccc}
\hline Soil distribution & $\begin{array}{c}\text { Layer thickness } \\
(\mathrm{m})\end{array}$ & $\begin{array}{c}\text { Case I } \\
\text { (Soft soil } \\
\text { covered) }\end{array}$ & $\begin{array}{c}\text { Case II } \\
\text { (Es increasing } \\
\text { with depth) }\end{array}$ & $\begin{array}{c}\text { Case III } \\
\text { (Stiff soil } \\
\text { covered) }\end{array}$ \\
\hline Layer 1 & 1 & 10000 & 3000 & 500 \\
Layer 2 & 1.5 & 2000 & 2000 & 2000 \\
Layer 3 & 2 & 1500 & 1500 & 1500 \\
Layer 4 & 3 & 1000 & 1000 & 1000 \\
Layer 5 & $\infty$ & 500 & 500 & 500 \\
\hline
\end{tabular}




\section{Appendix I}

$[\boldsymbol{t a}]_{i}=\left\{\boldsymbol{a}_{i}^{1} \boldsymbol{a}_{i}^{2} \boldsymbol{a}_{i}^{3} \boldsymbol{a}_{i}^{4}\right\}^{T} ;$

where

$$
\begin{aligned}
& \left\{\boldsymbol{a}_{i}^{1}\right\}=\left\{\cosh \left(\frac{\alpha_{i}}{h_{i}} z\right) \sinh \left(\frac{\alpha_{i}}{h_{i}} z\right) \cos \left(\frac{\beta_{i}}{h_{i}} z\right) \sin \left(\frac{\beta_{i}}{h_{i}} z\right)\right\}
\end{aligned}
$$

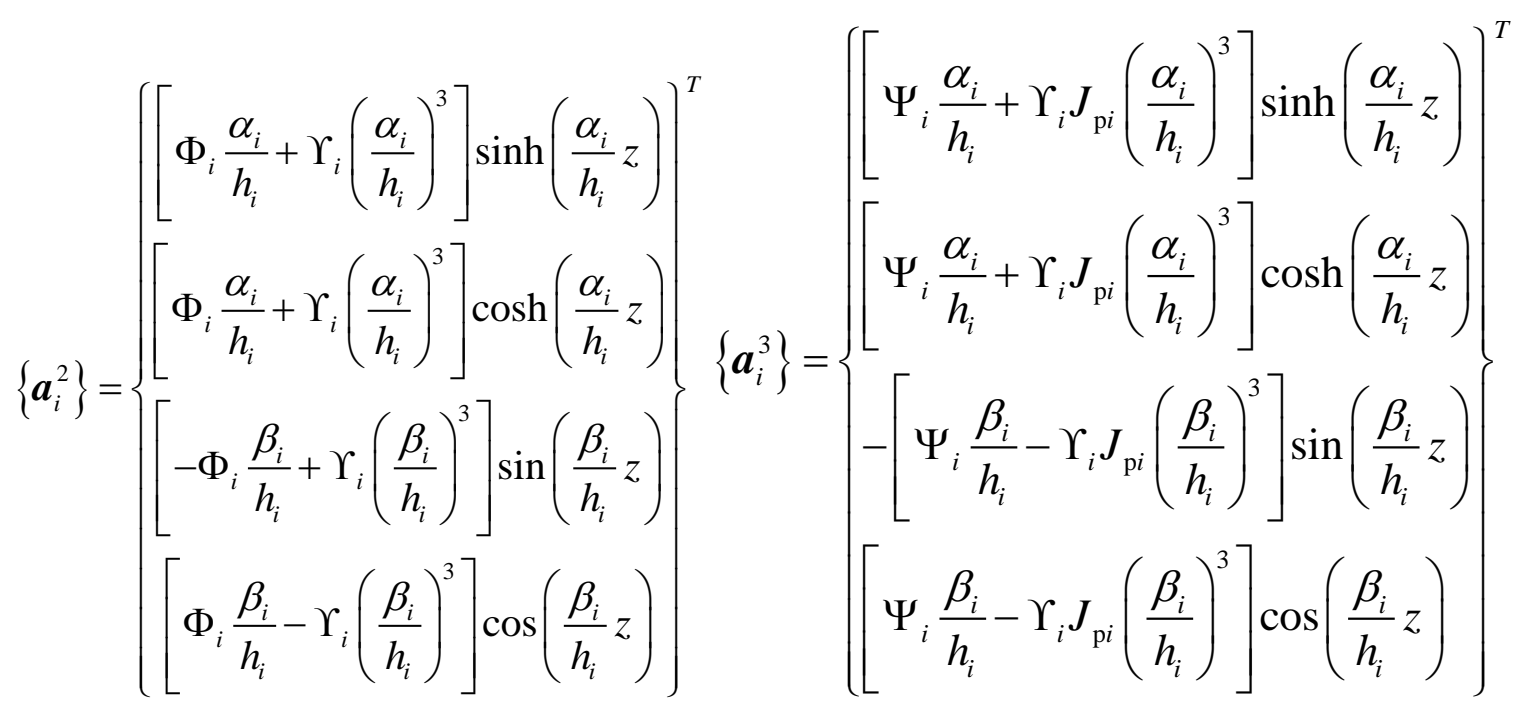

$$
\begin{aligned}
& \left.\int-E_{\mathrm{p} i} I_{\mathrm{p} i}\left[\Phi_{i}\left(\frac{\alpha_{i}}{h_{i}}\right)^{2}+\Upsilon_{i} J_{\mathrm{p} i}\left(\frac{\alpha_{i}}{h_{i}}\right)^{4}\right] \cosh \left(\frac{\alpha_{i}}{h_{i}} z\right)\right]^{T} \\
& \left\{\boldsymbol{a}_{i}^{4}\right\}=\left\{\begin{array}{l}
-E_{\mathrm{p} i} I_{\mathrm{p} i}\left[\Phi_{i}\left(\frac{\alpha_{i}}{h_{i}}\right)^{2}+\Upsilon_{i} J_{\mathrm{p} i}\left(\frac{\alpha_{i}}{h_{i}}\right)^{4}\right] \sinh \left(\frac{\alpha_{i}}{h_{i}} Z\right) \\
E_{\mathrm{p} i} I_{\mathrm{p} i}\left[\Phi_{i}\left(\frac{\beta_{i}}{h_{i}}\right)^{2}-\Upsilon_{i} J_{\mathrm{p} i}\left(\frac{\beta_{i}}{h_{i}}\right)^{4}\right] \cos \left(\frac{\beta_{i}}{h_{i}} Z\right) \\
E_{\mathrm{p} i} I_{\mathrm{p} i}\left[\Phi_{i}\left(\frac{\beta_{i}}{h_{i}}\right)^{2}-\Upsilon_{i} J_{\mathrm{p} i}\left(\frac{\beta_{i}}{h_{i}}\right)^{4}\right] \sin \left(\frac{\beta_{i}}{h_{i}} Z\right)
\end{array}\right\}
\end{aligned}
$$

with $\Phi_{i}=\frac{J_{\mathrm{p} i}}{J_{\mathrm{p} i}-W_{\mathrm{p} i}}+\frac{R_{i} E_{\mathrm{p} i} I_{\mathrm{p} i}}{J_{\mathrm{p} i}\left(J_{\mathrm{p} i}-W_{\mathrm{p} i}\right)}, \Psi_{i}=\frac{R_{i} E_{\mathrm{p} i} I_{\mathrm{p} i}+W_{\mathrm{p} i} J_{\mathrm{p} i}}{J_{\mathrm{p} i}-W_{\mathrm{p} i}}, \Upsilon_{i}=\frac{E_{\mathrm{p} i} I_{\mathrm{p} i}\left(J_{\mathrm{p} i}+g_{x i}\right)}{J_{\mathrm{p} i}\left(J_{\mathrm{p} i}-W_{\mathrm{p} i}\right)}$. 


\section{Appendix II}

$$
\begin{aligned}
& {[\boldsymbol{t b}]_{i}=\left\{\boldsymbol{b}_{i}^{1} \boldsymbol{b}_{i}^{2} \boldsymbol{b}_{i}^{3} \boldsymbol{b}_{i}^{4}\right\}^{T}} \\
& \left\{\boldsymbol{b}_{i}^{1}\right\}=\left\{z F_{a i} \sinh \left(\frac{\alpha_{i}}{h_{i}} z\right) \quad z F_{a i} \cosh \left(\frac{\alpha_{i}}{h_{i}} z\right) \quad-z F_{b i} \sin \left(\frac{\beta_{i}}{h_{i}} z\right) \quad z F_{b i} \cos \left(\frac{\alpha_{i}}{h_{i}} z\right)\right\} \\
& \int \frac{F_{a i}}{J_{\mathrm{p} i}\left(J_{\mathrm{p} i}-W_{\mathrm{p} i}\right)}\left\{\left(\frac{\alpha_{i}}{h_{i}}\right)\left[J_{\mathrm{p} i}{ }^{2}+R_{i} E_{\mathrm{p} i} I_{\mathrm{p} i}+\left(\frac{\alpha_{i}}{h_{i}}\right)^{2} E_{\mathrm{p} i} I_{\mathrm{p} i}\left(J_{\mathrm{p} i}+g_{x i}\right)\right] z \cosh \left(\frac{\alpha_{i}}{h_{i}} z\right)+\left[J_{\mathrm{p} i}{ }^{2}+R_{i} E_{\mathrm{p} i} I_{\mathrm{p} i}+3\left(\frac{\alpha_{i}}{h_{i}}\right)^{2} E_{\mathrm{p} i} I_{\mathrm{p} i}\left(J_{\mathrm{p} i}+g_{x i}\right)\right] \sinh \left(\frac{\alpha_{i}}{h_{i}} z\right)\right\} \\
& \left\{\boldsymbol{b}_{i}^{2}\right\}=\left\{\frac{F_{a i}}{J_{\mathrm{p} i}\left(J_{\mathrm{p} i}-W_{\mathrm{p} i}\right)}\left\{\left[J_{\mathrm{p} i}{ }^{2}+R_{i} E_{\mathrm{p} i} I_{\mathrm{p} i}+3\left(\frac{\alpha_{i}}{h_{i}}\right)^{2} E_{\mathrm{p} i} I_{\mathrm{p} i}\left(J_{\mathrm{p} i}+g_{x i}\right)\right] \cosh \left(\frac{\alpha_{i}}{h_{i}} z\right)+\left(\frac{\alpha_{1 i}}{h_{i}}\right)\left[J_{\mathrm{p} i}{ }^{2}+R_{i} E_{\mathrm{p} i} I_{\mathrm{p} i}+\left(\frac{\alpha_{i}}{h_{i}}\right)^{2} E_{\mathrm{p} i} I_{\mathrm{p} i}\left(J_{\mathrm{p} i}+g_{x i}\right)\right] z \sinh \left(\frac{\alpha_{i}}{h_{i}} z\right)\right\}\right\} \\
& -\frac{F_{b i}}{J_{\mathrm{p} i}\left(J_{\mathrm{p} i}-W_{\mathrm{p} i}\right)}\left\{\left(\frac{\beta_{i}}{h_{i}}\right)\left[J_{\mathrm{p} i}{ }^{2}+R_{i} E_{\mathrm{p} i} I_{\mathrm{p} i}-\left(\frac{\beta_{i}}{h_{i}}\right)^{2} E_{\mathrm{p} i} I_{\mathrm{p} i}\left(J_{\mathrm{p} i}+g_{x i}\right)\right] z \cos \left(\frac{\beta_{i}}{h_{i}} Z\right)+\left[J_{\mathrm{p} i}{ }^{2}+R_{i} E_{\mathrm{p} i} I_{\mathrm{p} i}-3\left(\frac{\beta_{i}}{h_{i}}\right)^{2} E_{\mathrm{p} i} I_{\mathrm{p} i}\left(J_{\mathrm{p} i}+g_{x i}\right)\right] \sin \left(\frac{\beta_{i}}{h_{i}} Z\right)\right\} \\
& \frac{F_{b i}}{J_{\mathrm{p} i}\left(J_{\mathrm{p} i}-W_{\mathrm{p} i}\right)}\left\{\left[J_{\mathrm{p} i}{ }^{2}+R_{i} E_{\mathrm{p} i} I_{\mathrm{p} i}-3\left(\frac{\beta_{i}}{h_{i}}\right)^{2} E_{\mathrm{p} i} I_{\mathrm{p} i}\left(J_{\mathrm{p} i}+g_{x i}\right)\right] \cos \left(\frac{\beta_{i}}{h_{i}} z\right)-\left(\frac{\beta_{i}}{h_{i}}\right)\left[J_{\mathrm{p} i}{ }^{2}+R_{i} E_{\mathrm{p} i} I_{\mathrm{p} i}-\left(\frac{\beta_{i}}{h_{i}}\right)^{2} E_{\mathrm{p} i} I_{\mathrm{p} i}\left(J_{\mathrm{p} i}+g_{x i}\right)\right] z \sin \left(\frac{\beta_{i}}{h_{i}} z\right)\right\} \\
& \int \frac{F_{a i}}{J_{\mathrm{p} i}-W_{\mathrm{p} i}}\left\{\left(\frac{\alpha_{i}}{h_{i}}\right)\left[R_{i} E_{\mathrm{p} i} I_{\mathrm{p} i}+J_{\mathrm{p} i} W_{\mathrm{p} i}+\left(\frac{\alpha_{i}}{h_{i}}\right)^{2} E_{\mathrm{p} i} I_{\mathrm{p} i}\left(J_{\mathrm{p} i}+g_{x i}\right)\right] z \cosh \left(\frac{\alpha_{i}}{h_{i}} z\right)+\left[R_{i} E_{\mathrm{p} i} I_{\mathrm{p} i}+J_{\mathrm{p} i} W_{\mathrm{p} i}+3\left(\frac{\alpha_{i}}{h_{i}}\right)^{2} E_{\mathrm{p} i} I_{\mathrm{p} i}\left(J_{\mathrm{p} i}+g_{x i}\right)\right] \sinh \left(\frac{\alpha_{i}}{h_{i}} Z\right)\right\} \\
& \left\{\boldsymbol{b}_{i}^{3}\right\}=\left\{\frac{F_{a i}}{J_{\mathrm{p} i}-W_{\mathrm{p} i}}\left\{\left[R_{i} E_{\mathrm{p} i} I_{\mathrm{p} i}+J_{\mathrm{p} i} W_{\mathrm{p} i}+3\left(\frac{\alpha_{i}}{h_{i}}\right)^{2} E_{\mathrm{p} i} I_{\mathrm{p} i}\left(J_{\mathrm{p} i}+g_{x i}\right)\right] \cosh \left(\frac{\alpha_{i}}{h_{i}} z\right)+\left(\frac{\alpha_{i}}{h_{i}}\right)\left[R_{i} E_{\mathrm{p} i} I_{\mathrm{p} i}+J_{\mathrm{p} i} W_{\mathrm{p} i}+\left(\frac{\alpha_{i}}{h_{i}}\right)^{2} E_{\mathrm{p} i} I_{\mathrm{p} i}\left(J_{\mathrm{p} i}+g_{x i}\right)\right] z \sinh \left(\frac{\alpha_{i}}{h_{i}} z\right)\right\}\right\} \\
& -\frac{F_{b i}}{J_{\mathrm{p} i}-W_{\mathrm{p} i}}\left\{\left(\frac{\beta_{i}}{h_{\mathrm{i}}}\right)\left[R_{i} E_{\mathrm{p} i} I_{\mathrm{p} i}+J_{\mathrm{p} i} W_{\mathrm{p} i}-\left(\frac{\beta_{i}}{h_{i}}\right)^{2} E_{\mathrm{p} i} I_{\mathrm{p} i}\left(J_{\mathrm{p} i}+g_{x i}\right)\right] z \cos \left(\frac{\beta_{i}}{h_{i}} Z\right)+\left[R_{i} E_{\mathrm{p} i} I_{\mathrm{p} i}+J_{\mathrm{p} i} W_{\mathrm{p} i}-3\left(\frac{\beta_{i}}{h_{i}}\right)^{2} E_{\mathrm{p} i} I_{\mathrm{p} i}\left(J_{\mathrm{p} i}+g_{x i}\right)\right] \sin \left(\frac{\beta_{i}}{h_{i}} Z\right)\right\} \\
& \frac{F_{b i}}{J_{\mathrm{p} i}-W_{\mathrm{p} i}}\left\{\left[R_{i} E_{\mathrm{p} i} I_{\mathrm{p} i}+J_{\mathrm{p} i} W_{\mathrm{p} i}-3\left(\frac{\beta_{i}}{h_{i}}\right)^{2} E_{\mathrm{p} i} I_{\mathrm{p} i}\left(J_{\mathrm{p} i}+g_{x i}\right)\right] \cos \left(\frac{\beta_{i}}{h_{i}} z\right)-\left(\frac{\beta_{i}}{h_{i}}\right)\left[R_{i} E_{\mathrm{p} i} I_{\mathrm{p} i}+J_{\mathrm{p} i} W_{\mathrm{p} i}-\left(\frac{\beta_{i}}{h_{i}}\right)^{2} E_{\mathrm{p} i} I_{\mathrm{p} i}\left(J_{\mathrm{p} i}+g_{x i}\right)\right] z \sin \left(\frac{\beta_{i}}{h_{i}} z\right)\right\}
\end{aligned}
$$




$$
\begin{aligned}
& \left.\int-\frac{F_{a i} E_{\mathrm{pi}} I_{\mathrm{pi}}}{J_{\mathrm{p} i}\left(J_{\mathrm{p} i}-W_{\mathrm{pi}}\right)}\left(\frac{\alpha_{i}}{h_{i}}\right)\left\{2\left[J_{\mathrm{pi}}{ }^{2}+R_{i} E_{\mathrm{pi}} I_{\mathrm{pi}}+2\left(\frac{\alpha_{i}}{h_{i}}\right)^{2} E_{\mathrm{pi}} I_{\mathrm{pi}}\left(J_{\mathrm{pi}}+g_{x i}\right)\right] \cosh \left(\frac{\alpha_{i}}{h_{i}} z\right)+\left(\frac{\alpha_{i}}{h_{i}}\right)\left[J_{\mathrm{pi}}{ }^{2}+R_{i} E_{\mathrm{pi}} I_{\mathrm{pi}}+\left(\frac{\alpha_{i}}{h_{i}}\right)^{2} E_{\mathrm{pi}} I_{\mathrm{p} i}\left(J_{\mathrm{pi}}+g_{x i}\right)\right] z \sinh \left(\frac{\alpha_{i}}{h_{i}} z\right)\right\}\right) \\
& \left\{\boldsymbol{b}_{i}^{4}\right\}=\left\{-\frac{F_{a i} E_{\mathrm{p} i} I_{\mathrm{p} i}}{J_{\mathrm{p} i}\left(J_{\mathrm{pi}}-W_{\mathrm{p} i}\right.}\left(\frac{\alpha_{i}}{h_{i}}\right)\left\{\left(\frac{\alpha_{i}}{h_{i}}\right)\left[J_{\mathrm{pi}}{ }^{2}+R_{i} E_{\mathrm{pi}} I_{\mathrm{pi}}+\left(\frac{\alpha_{i}}{h_{i}}\right)^{2} E_{\mathrm{p} i} I_{\mathrm{p} i}\left(J_{\mathrm{p} i}+g_{x i}\right)\right] z \cosh \left(\frac{\alpha_{i}}{h_{i}} z\right)+2\left[J_{\mathrm{p} i}{ }^{2}+R_{i} E_{\mathrm{pi}} I_{\mathrm{pi}}+2\left(\frac{\alpha_{i}}{h_{i}}\right)^{2} E_{\mathrm{pi}} I_{\mathrm{pi}}\left(J_{\mathrm{p} i}+g_{x i}\right)\right] \sinh \left(\frac{\alpha_{i}}{h_{i}} Z\right)\right\}\right. \\
& \left\{\frac{F_{b i} E_{\mathrm{p} i} I_{\mathrm{pi}}}{J_{\mathrm{p} i}\left(J_{\mathrm{p} i}-W_{\mathrm{p} i}\right.}\left(\frac{\beta_{i}}{h_{i}}\right)\left\{2\left[J_{\mathrm{p} i}{ }^{2}+R_{i} E_{\mathrm{p} i} I_{\mathrm{pi}}-2\left(\frac{\beta_{i}}{h_{i}}\right)^{2} E_{\mathrm{pi}} I_{\mathrm{p} i}\left(J_{\mathrm{p} i}+g_{x i}\right)\right] \cos \left(\frac{\beta_{i}}{h_{i}} z\right)-\left(\frac{\beta_{i}}{h_{i}}\right)\left[J_{\mathrm{pi}}{ }^{2}+R_{i} E_{\mathrm{pi}} I_{\mathrm{pi}}-\left(\frac{\beta_{i}}{h_{i}}\right)^{2} E_{\mathrm{p} i} I_{\mathrm{pi}}\left(J_{\mathrm{p} i}+g_{x i}\right)\right] z \sin \left(\frac{\beta_{i}}{h_{i}} z\right)\right\}\right. \\
& \frac{F_{\mathrm{bi}} E_{\mathrm{pi}} I_{\mathrm{pi}}}{J_{\mathrm{p} i}\left(J_{\mathrm{pi} i}-W_{\mathrm{pi}}\right)}\left(\frac{\beta_{i}}{h_{i}}\right)\left\{\left(\frac{\beta_{i}}{h_{i}}\right)\left[J_{\mathrm{pi}}{ }^{2}+R_{i} E_{\mathrm{pi}} I_{\mathrm{pi}}-\left(\frac{\beta_{i}}{h_{i}}\right)^{2} E_{\mathrm{pi}} I_{\mathrm{pi}}\left(J_{\mathrm{p} i}+g_{\mathrm{x} i}\right)\right] z \cos \left(\frac{\beta_{i}}{h_{i}} z\right)+2\left[J_{\mathrm{pi}}{ }^{2}+R_{i} E_{\mathrm{pi}} I_{\mathrm{pi}}-2\left(\frac{\beta_{i}}{h_{i}}\right)^{2} E_{\mathrm{pi}} I_{\mathrm{pi}}\left(J_{\mathrm{pi}}+g_{x i}\right)\right] \sin \left(\frac{\beta_{i}}{h_{i}} z\right)\right\}
\end{aligned}
$$




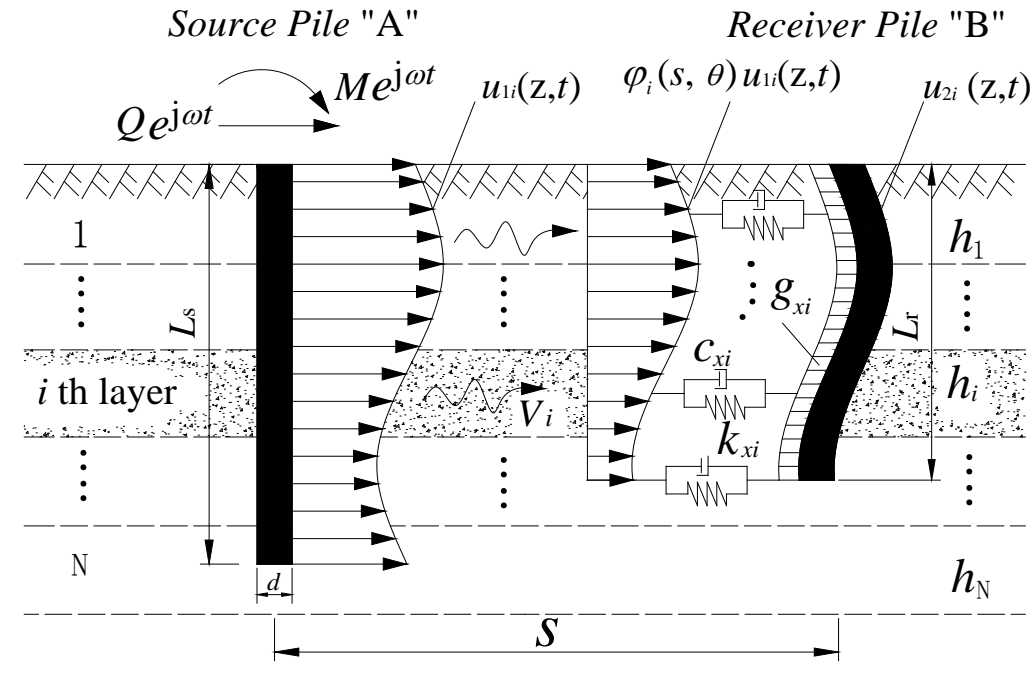

(a)

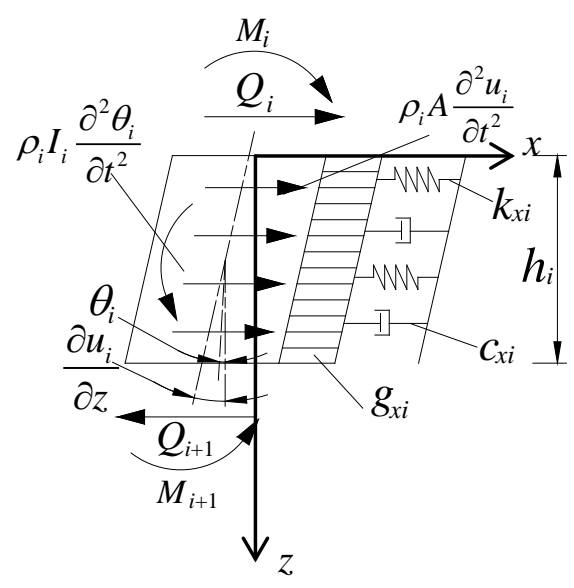

(b)

Fig 1. Model description:(a) pile-to-pile dynamic interaction in the Pasternak layered foundation; (b) deflected differential layered-pile element

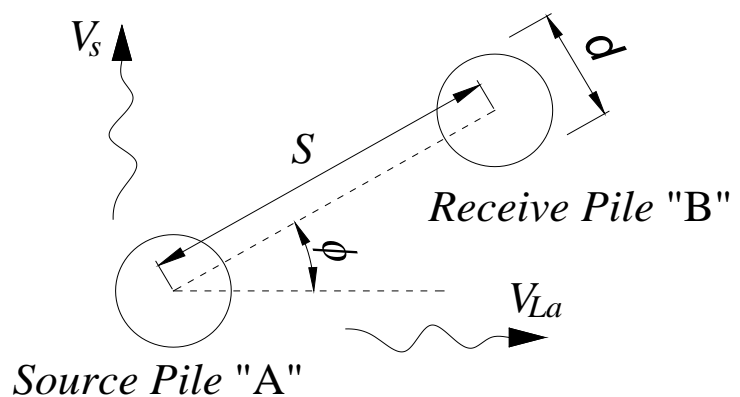

Fig.2 Relative position of two piles 

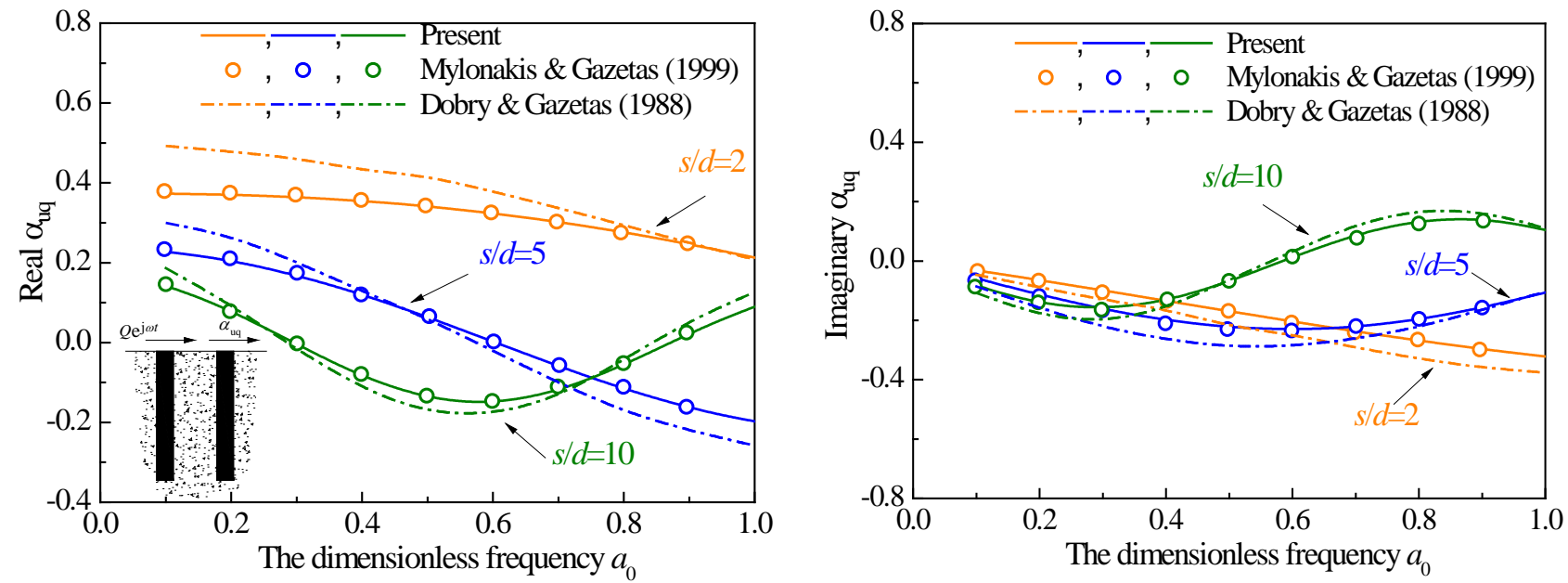

(a) Lateral interaction factor
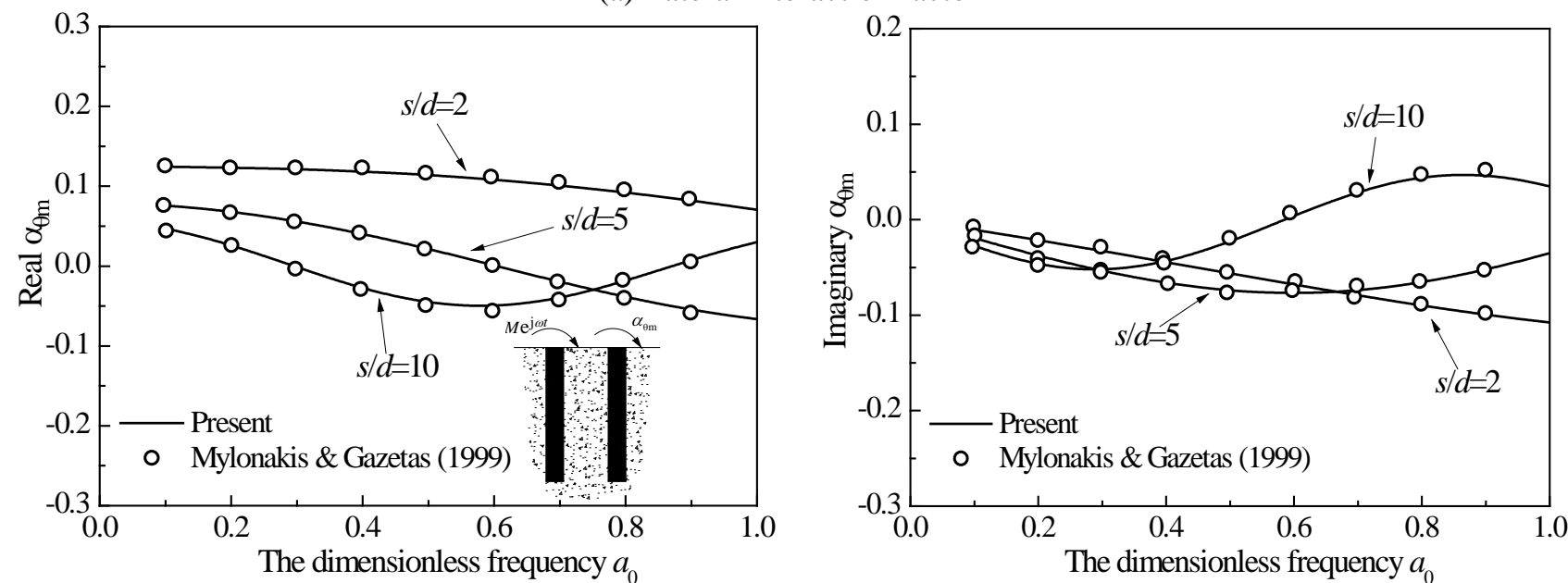

(b) Rocking interaction factor

Fig. 3 Dynamic interaction factors between present analysis with other solutions in homogeneous half-space $\left(\beta_{\mathrm{s}}=5 \%, v_{\mathrm{s}}=0.4, v_{p}=0.3, L / d=20, \mathrm{~s} / d=2, E_{\mathrm{p}} / E_{\mathrm{s}}=1000, \rho_{\mathrm{p}} / \rho_{\mathrm{s}}=1.5, a_{0}=\omega d / V_{\mathrm{s}}\right)$
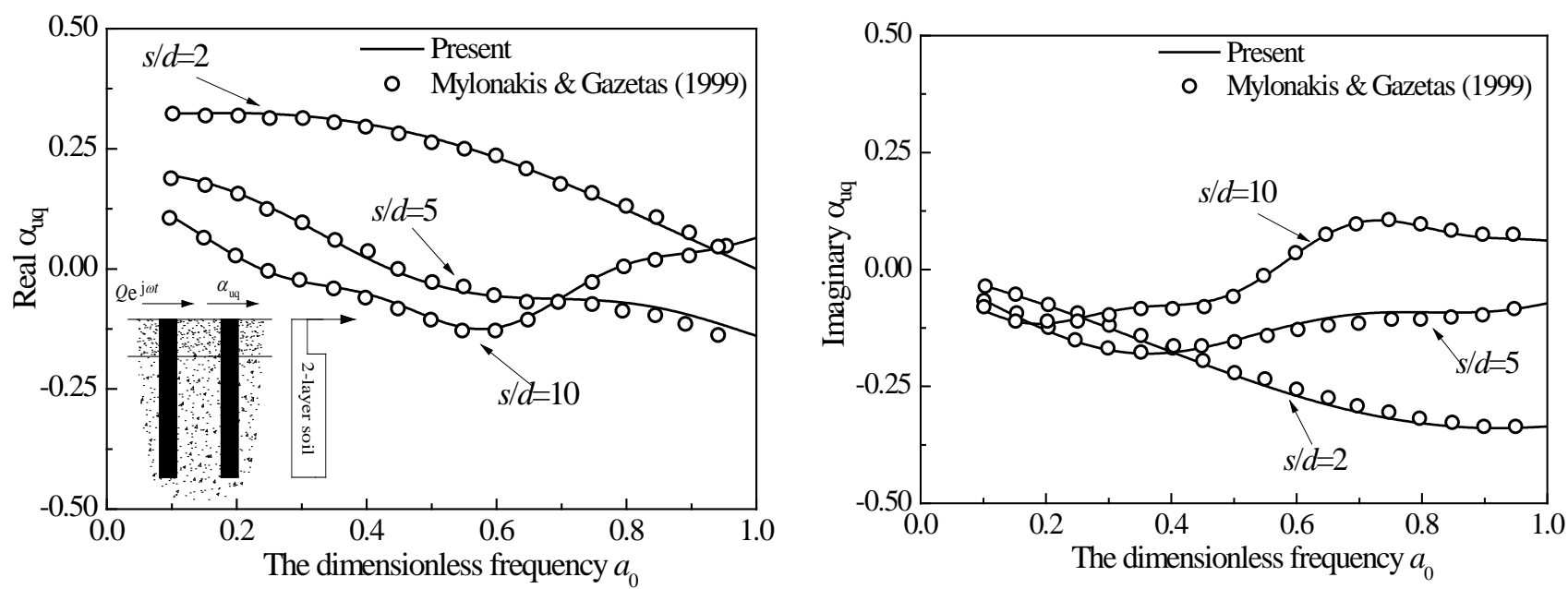

Fig. 4 Lateral dynamic interaction factors between present analysis with other solutions in half-space overlain by soft layer with $E_{\mathrm{p}} / E_{\mathrm{s} 1}=10000$ and $h_{1} / d=1$

$\left(\beta_{\mathrm{s}}=5 \%, v_{\mathrm{s}}=0.4, v_{p}=0.3, L / d=20, E_{\mathrm{p}} / E_{\mathrm{s} 2}=1000, \rho_{\mathrm{p}} / \rho_{\mathrm{s}}=1.3, a_{0}=\omega d / V_{\mathrm{s} 2}\right)$ 

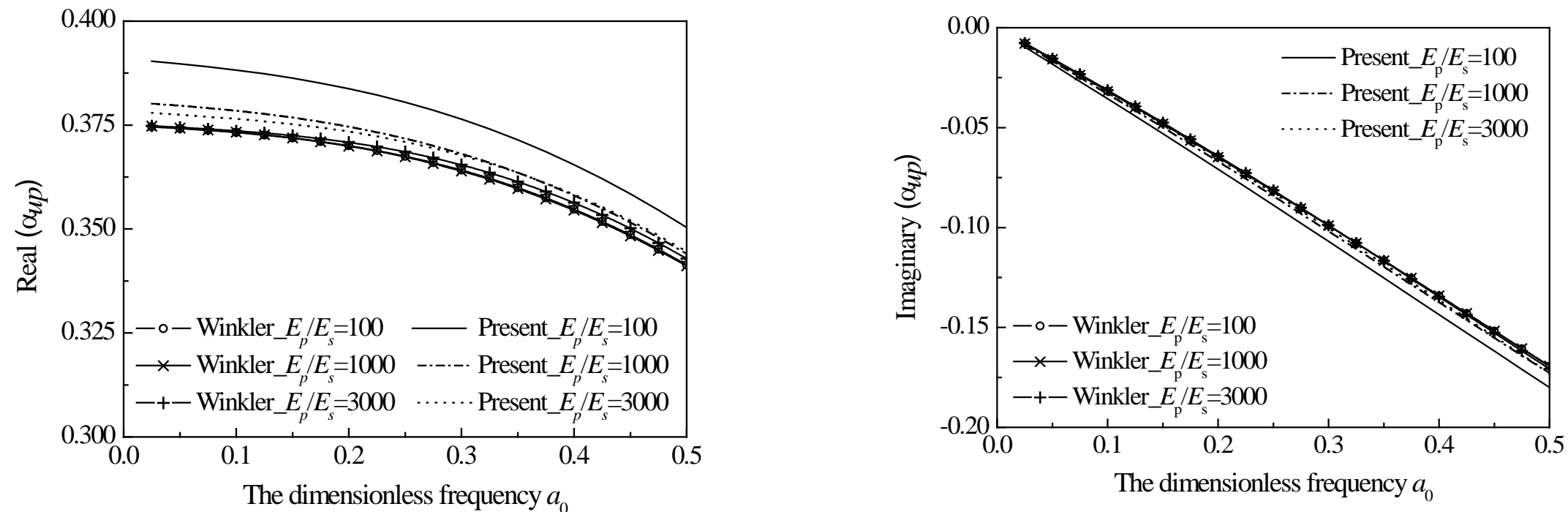

(a) Lateral dynamic interaction factor
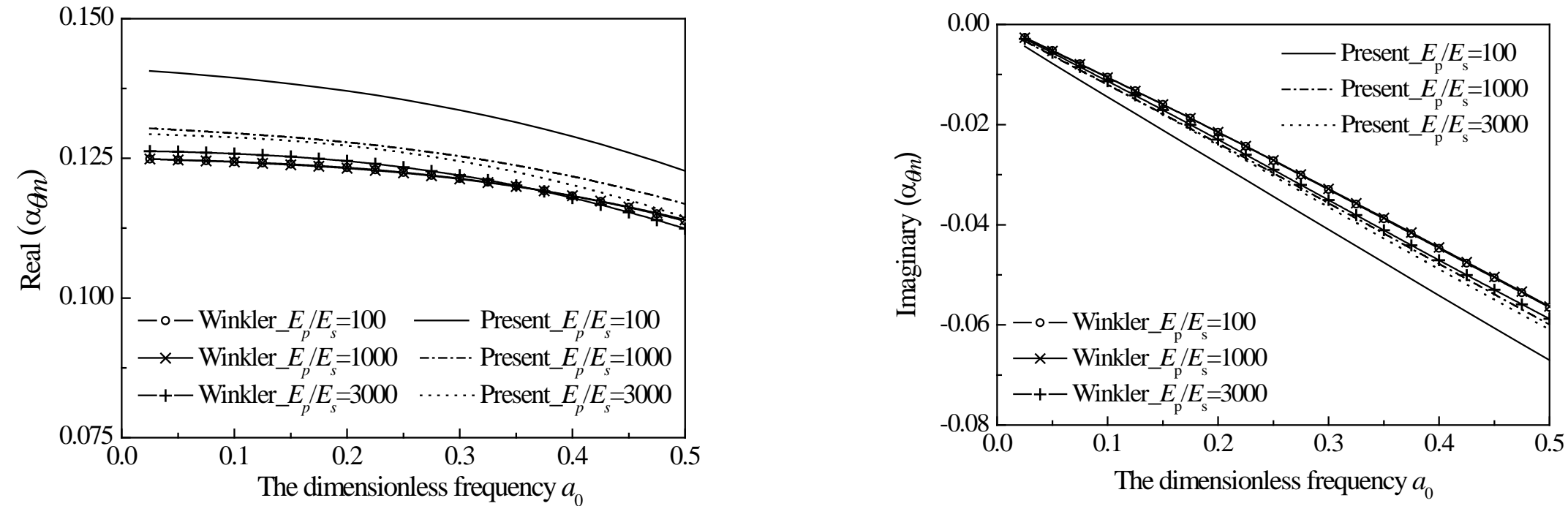

(b) Rocking dynamic interaction factor

Fig. 5 Shear effect of soil for interaction factor with different pile-soil modulus ratio $\left(\beta_{s}=5 \%, v_{s}=0.4, v_{p}=0.3, L / d=20, s / d=2, a_{0}=\omega d / V_{s}\right)$ 


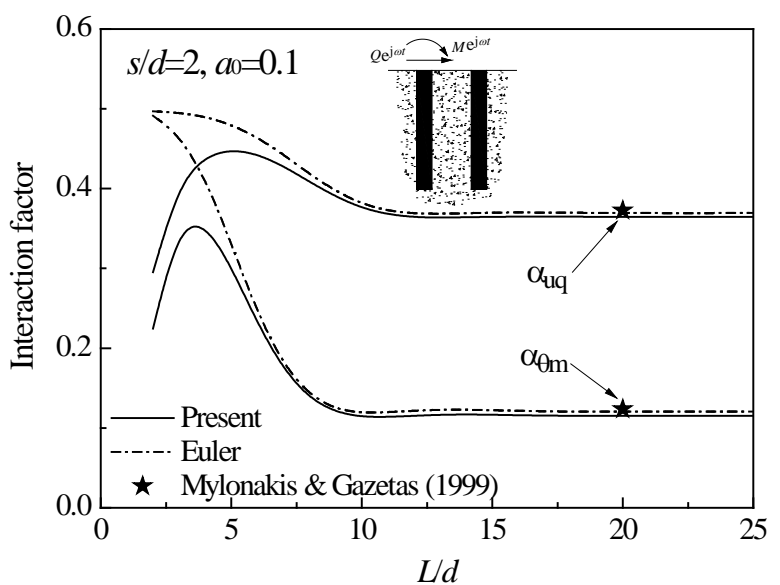

(a)

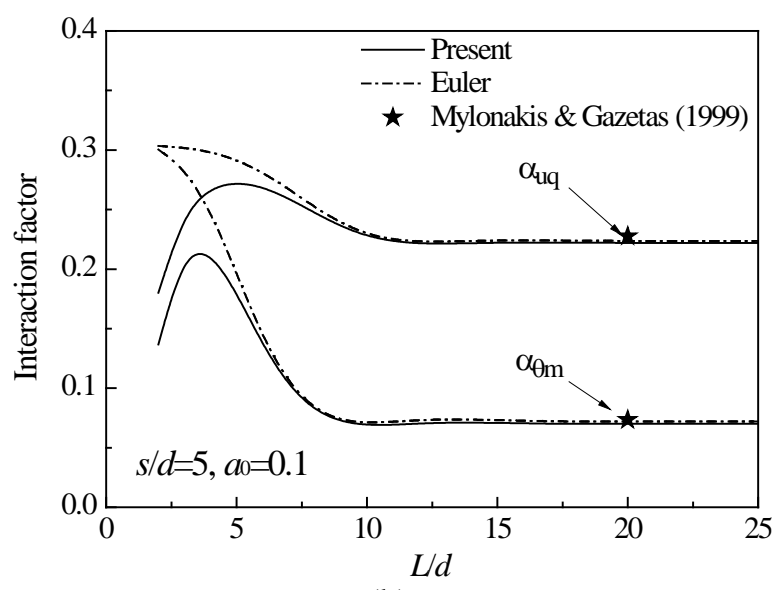

(b)

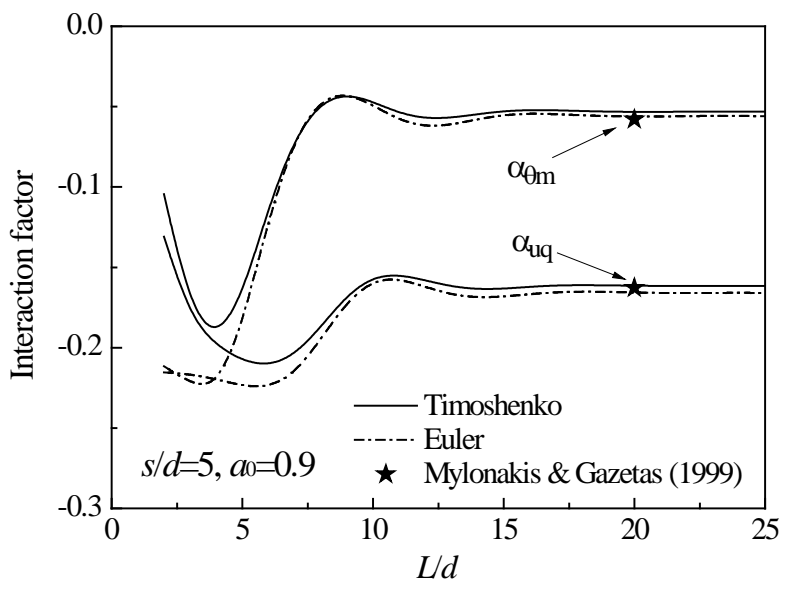

(c)

Fig. 6 Shear effect of piles in homogenous half-space $\left(\beta_{\mathrm{s}}=5 \%, v_{\mathrm{s}}=0.4, v_{p}=0.3, L / d=20, \mathrm{~s} / \mathrm{d}=2, E_{\mathrm{p}} / E_{\mathrm{s}}=1000, \rho_{\mathrm{p}} / \rho_{\mathrm{s}}=1.5, a_{0}=\omega \mathrm{d} / V_{\mathrm{s}}\right)$

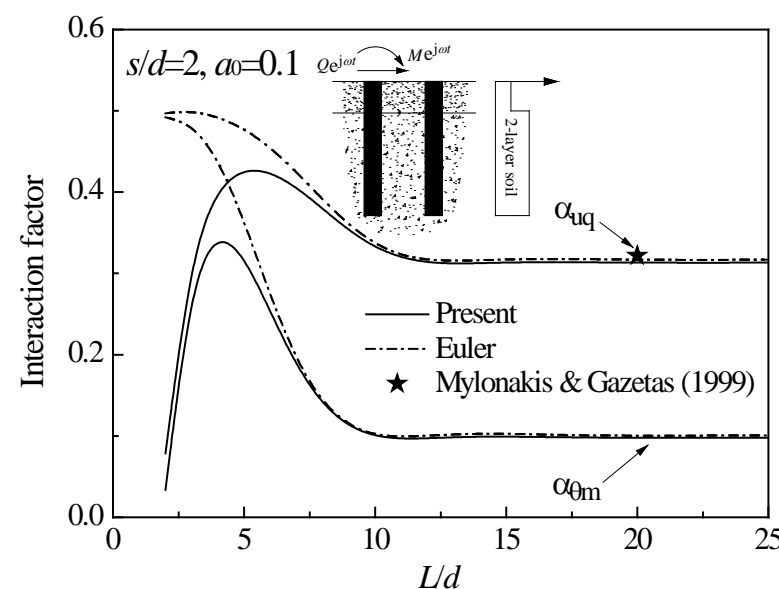

(a)

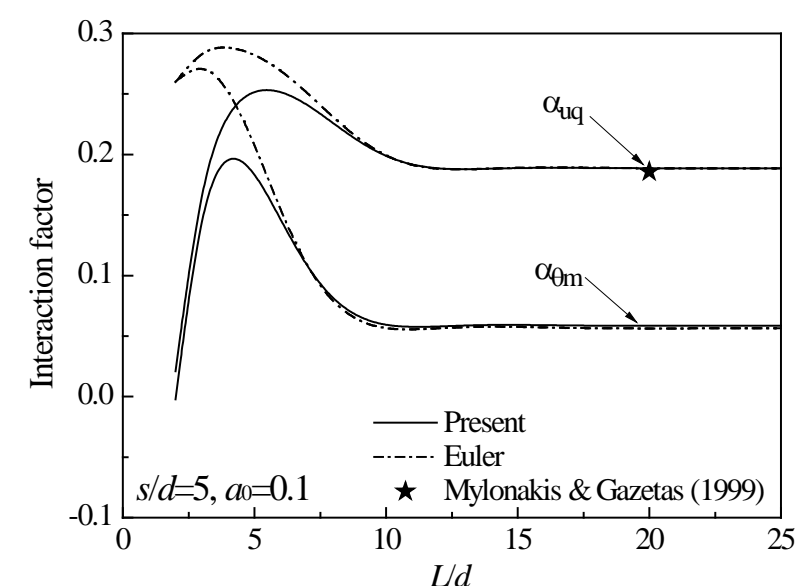

(b)

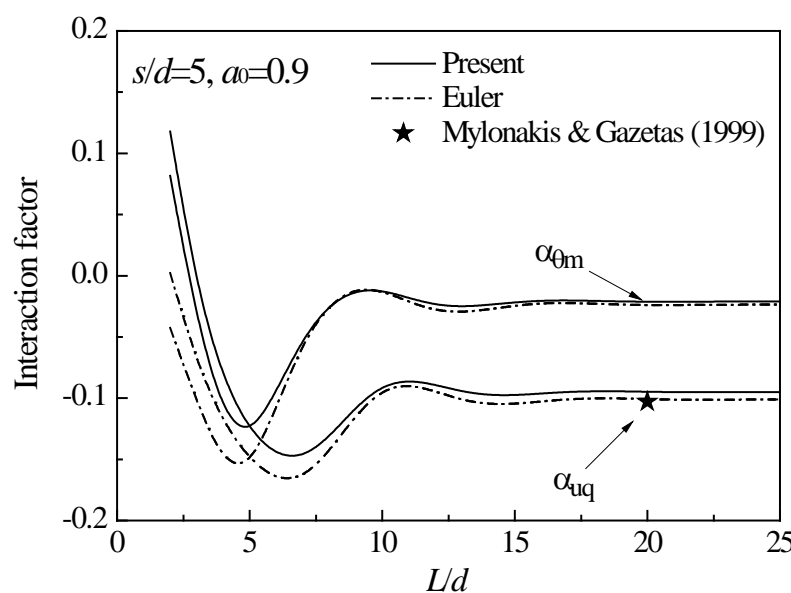

(c)

Fig. 7 Shear effect of piles in half-space overlain by soft layer with $E_{\mathrm{p}} / E_{\mathrm{s} 1}=10000$ and $h_{1} / d=1$ $\left(\beta_{\mathrm{s}}=5 \%, v_{\mathrm{s}}=0.4, v_{p}=0.3, L / d=20, E_{\mathrm{p}} / E_{\mathrm{s} 2}=1000, \rho_{\mathrm{p}} / \rho_{\mathrm{s}}=1.3, a_{0}=\omega d / V_{\mathrm{s} 2}\right)$ 
(a)

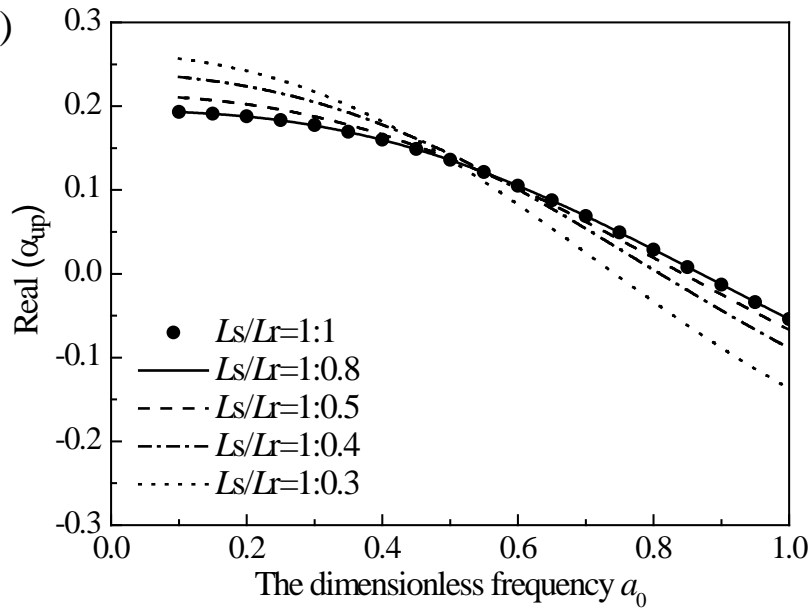

(b)

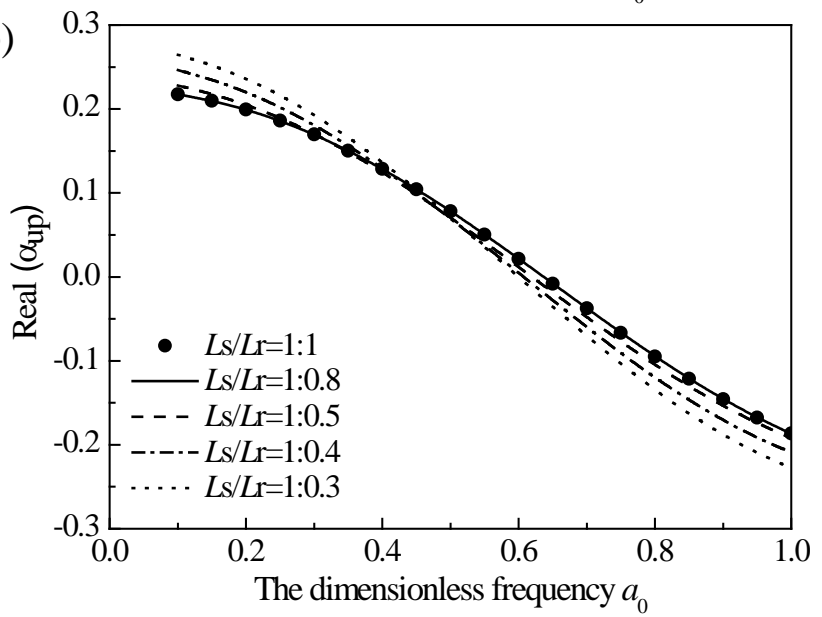

(c)

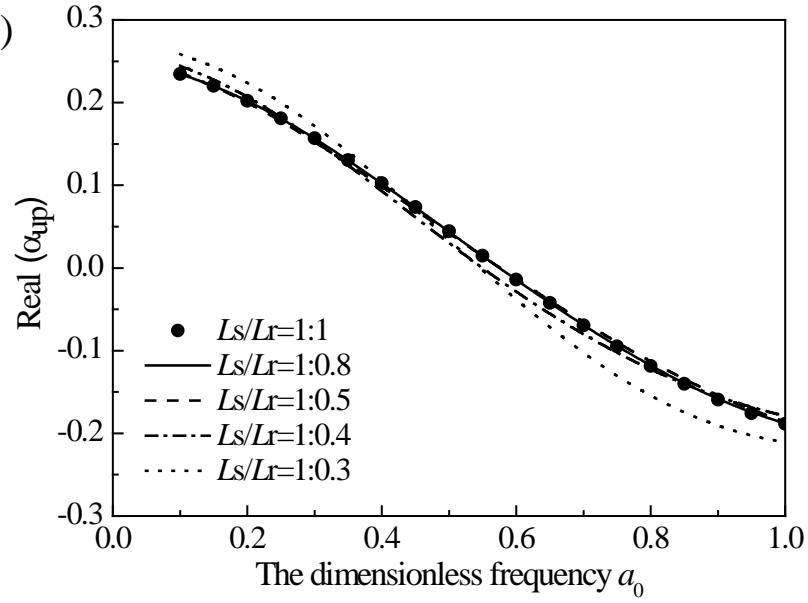

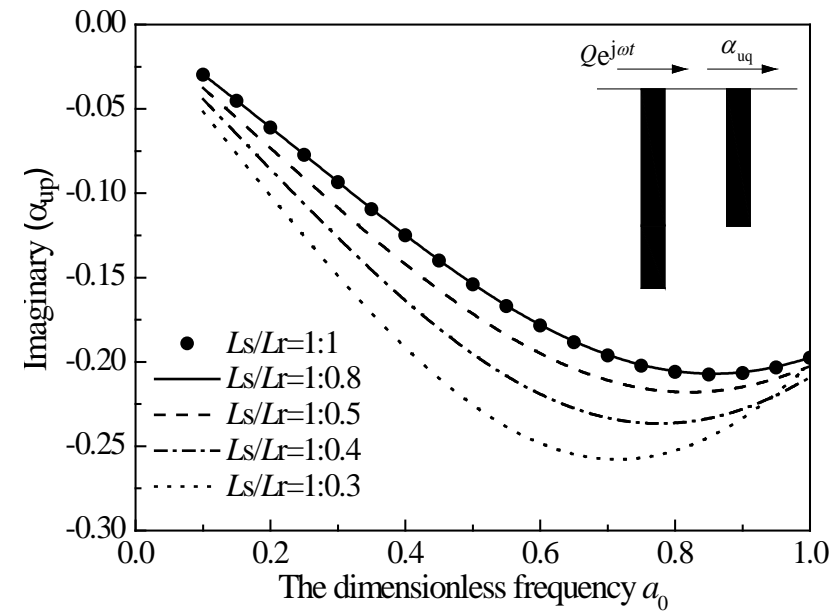
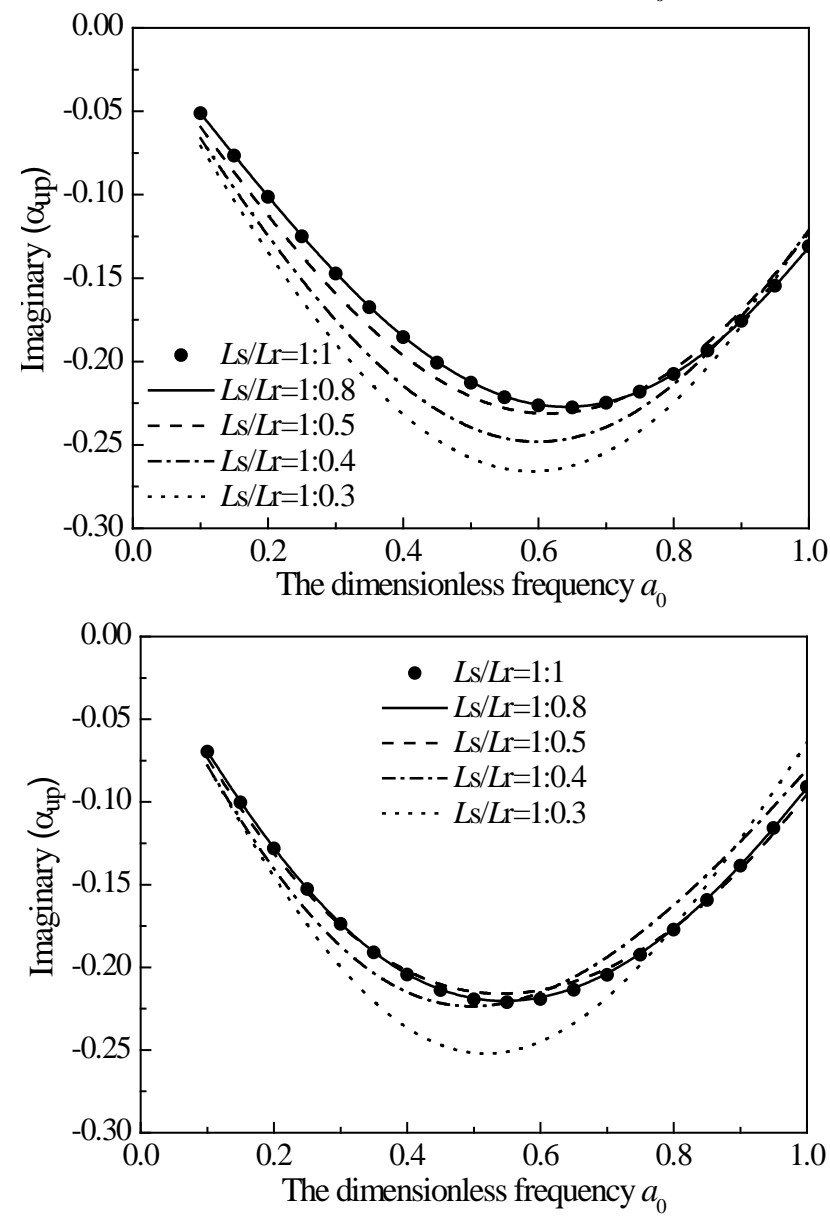

Fig.8 Variation of lateral interaction factors between piles with dissimilar $L_{\mathrm{s}} / L_{\mathrm{r}}$ under different soil foundation (a) Case 1, (b) Case 2, (c) Case 3

$\left(\beta_{\mathrm{s}}=5 \%, v_{\mathrm{s}}=0.4, v_{p}=0.3, L_{\mathrm{s}} / d=20, \mathrm{~s} / d=5, \rho_{\mathrm{p}} / \rho_{\mathrm{s}}=1.5, a_{0}=\omega d / V_{\mathrm{s} 1}\right)$ 
(a)

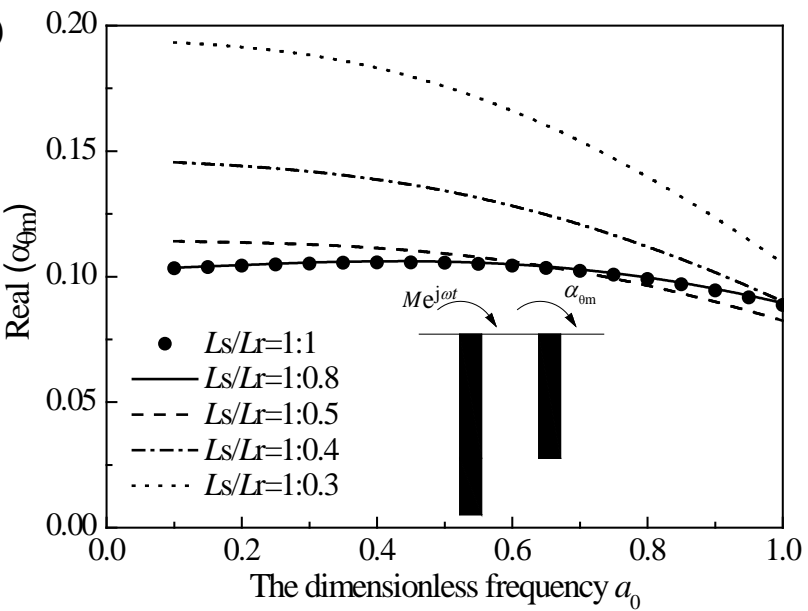

(b)

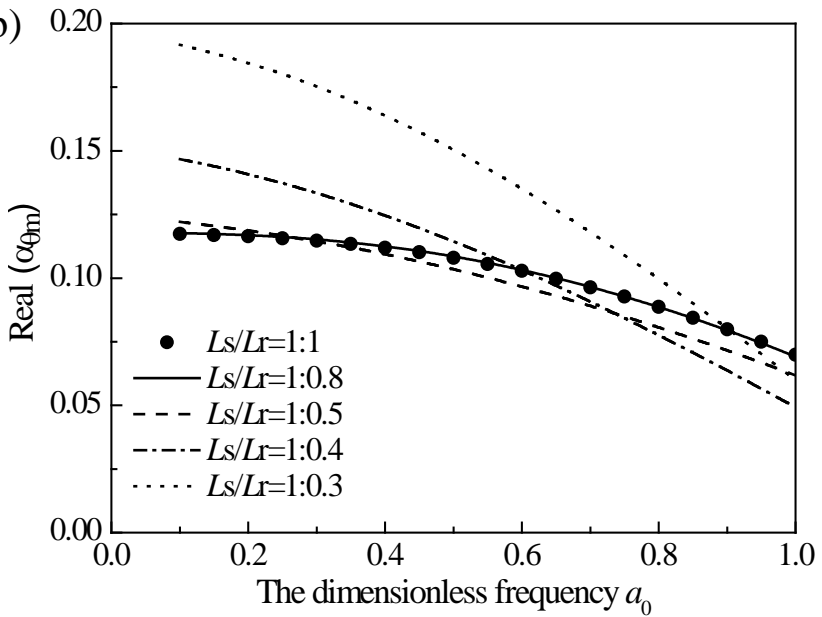

(c)

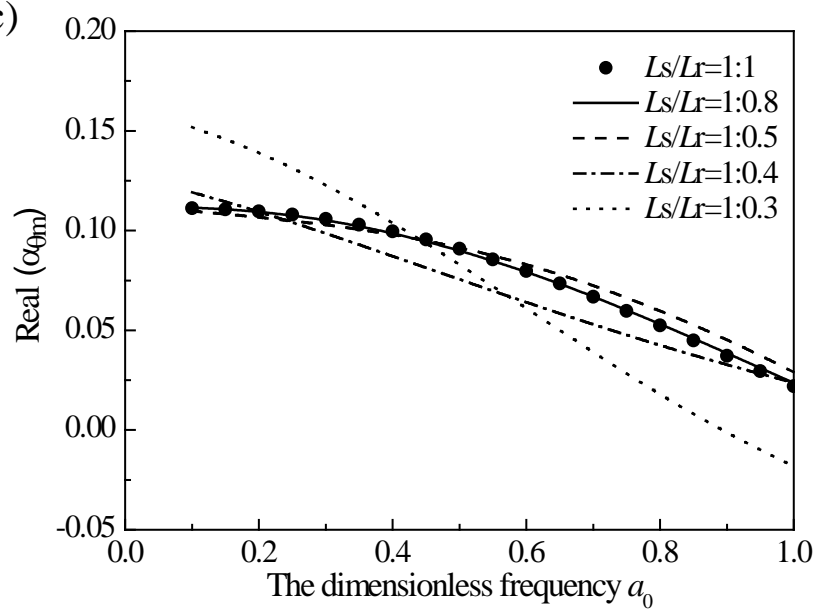

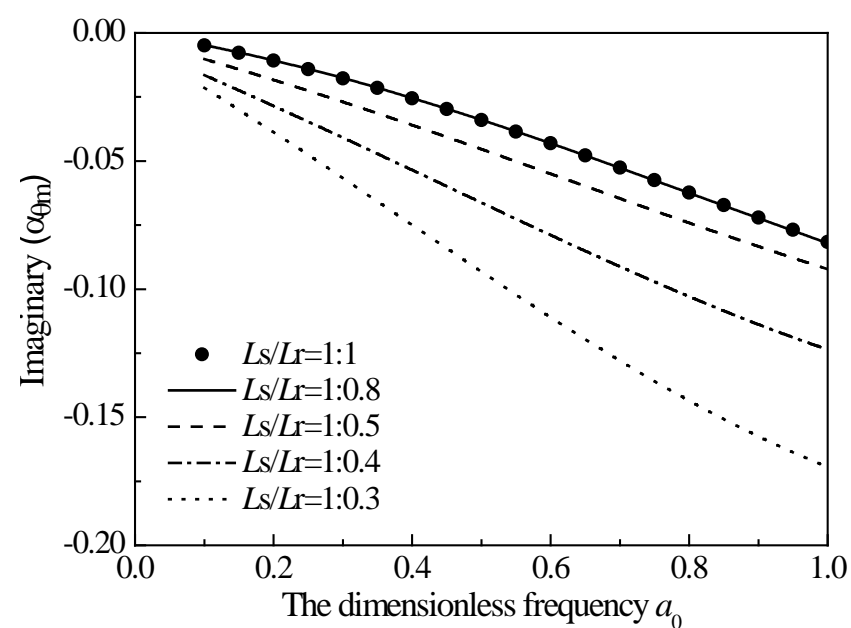
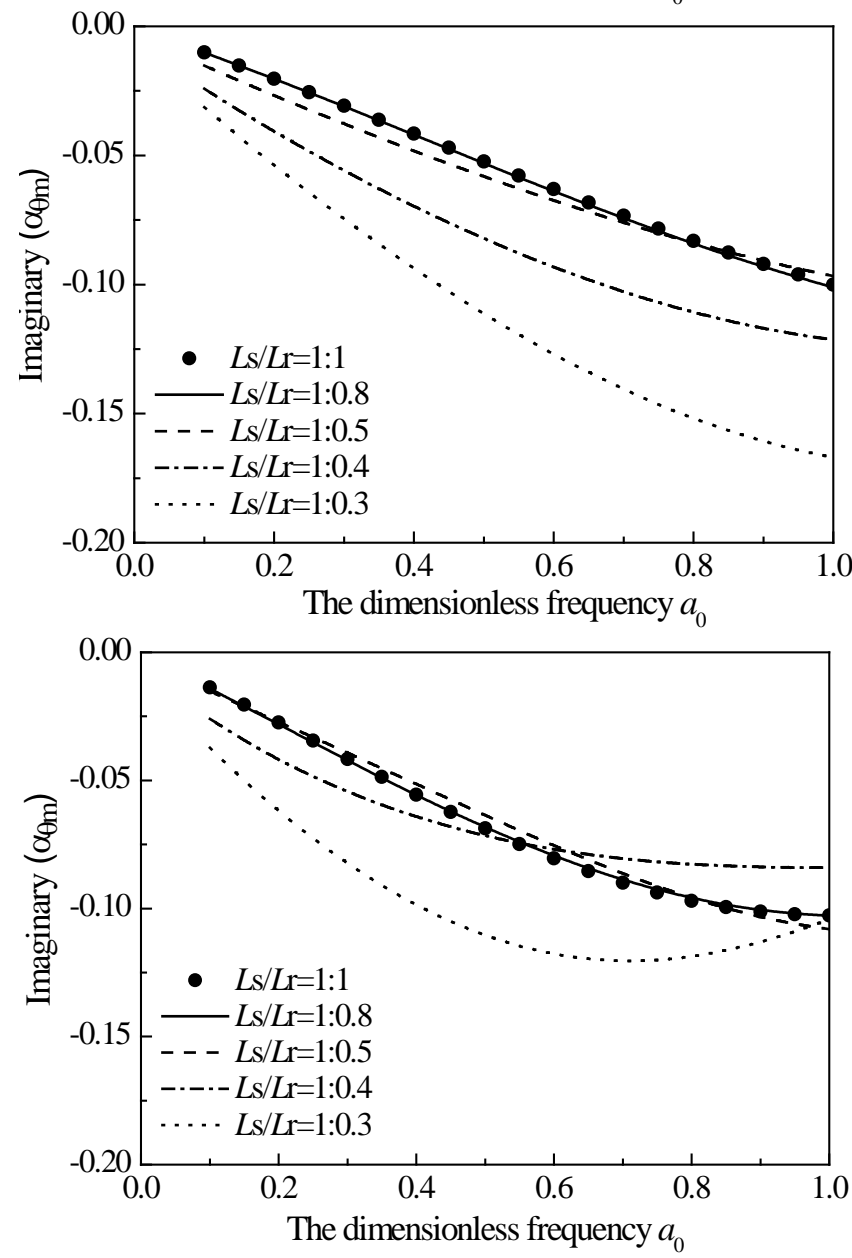

Fig.9 Variation of rocking interaction factors between piles with dissimilar $L_{\mathrm{s}} / L_{\mathrm{r}}$ under different soil foundation (a) Case 1, (b) Case 2, (c) Case 3

$\left(\beta_{\mathrm{s}}=5 \%, v_{\mathrm{s}}=0.4, v_{p}=0.3, L_{\mathrm{s}} / d=20, s / d=2, \rho_{\mathrm{p}} / \rho_{\mathrm{s}}=1.5, a_{0}=\omega \mathrm{d} / V_{\mathrm{s} 1}\right)$ 